\title{
International Conference on Lymphocyte Engineering September 13-15, 2019 \\ London, United Kingdom
}




\section{ICLE 2019 - Oral Presentations}

\section{ICLEP19-0001}

Allogeneic Act

\section{COMBINING INNATE IMMUNE TARGETING WITH ADAPTIVE IMMUNE POWER: EXPLOITING NKG2D FOR AUTOLOGOUS AND ALLOGENEIC CAR T CELL THERAPY}

D. Gilham ${ }^{1}$

Celyad S.A., Mont-Saint-Guibert, Belgium

The Natural Killer Group 2D (NKG2D) receptor enables natural killer cells to target infected cells through the multi-ligand specificity of NKG2D. The short life span of NK cells coupled with inherent inhibition of effector function through HLA expression on target cells suggests that NK cells may be restricted in their ability to engage tumor cells that readily express NKG2D ligands. Fusing NKG2D to the $\mathrm{CD} 3 \mathrm{z}$ intracellular domain generates a chimeric antigen receptor that endows the CAR T with specificity for the eight NKG2D ligands which are over-expressed on most solid tumors and hematological malignances. Initial clinical testing of the autologous NKG2D CAR T cells CYAD-01 using three infusions of cells given in the absence of pre-conditioning chemotherapy has achieved clinical responses in patients with relapsed/refractory Acute Myeloid Leukemia. Further clinical trials of CYAD-01 are on-going in combination with preconditioning chemotherapy. Moreover, CYAD-01 is also in clinical testing in patients with advanced metastatic colorectal cancer demonstrating the generic nature of NKG2D CAR T cell therapy.

In addition, our latest activity involves allogeneic CAR $\mathrm{T}$ cell therapy. The first product is CYAD-101 which uses the same NKG2D CAR combined with a non-gene edited allogeneic approach. We have recently reported the first two cohorts of metastatic colorectal patients who received the CYAD-101 combined with Folfox chemotherapy. Highly encouragingly, there was no evidence of symptoms of graft versus host disease which supports the move into higher cell doses on this trial. Celyad is further developing a non-gene edited allogeneic based upon shRNA technology that has potential application for any CAR T target.

\section{ICLEp19-0002}

\section{Allogeneic Act}

\section{GENETIC ENGINEERING OF OFF-THE-SHELF CAR T CELLS USING ARCUS NUCLEASES}

D. MacLeod $^{1}$, Aaron Martin ${ }^{1}$, Mark Johnson ${ }^{1}$, JoAnn Hux ${ }^{1}$, Jason Lee ${ }^{1}$, Michelle Pires ${ }^{1}$, Jeff Smith ${ }^{1}$, Bruce McCreedy ${ }^{1}$, Derek Jantz ${ }^{1}$

\section{${ }^{1}$ Precision BioSciences, Inc., Durham, NC, USA}

Gene-editing can be used to generate off-the-shelf allogeneic chimeric antigen receptor (CAR) T cell products and to impart a number of desirable features to these cells to improve their function. We have built a robust and scalable platform for the production of off-the-shelf gene-edited allogeneic CAR T cells, and are currently treating patients in a Phase I trial with a CD19targeted CAR T therapy for patients suffering with non-Hodgkin lymphoma and B-cell acute lymphoblastic leukemia. For our next generation of therapeutics, we are exploring additional modifications to improve allogeneic CAR $\mathrm{T}$ cell function, including incorporation of an RNAi cassette to reduce surface expression of major histocompatibility complex (MHC) class I molecules and potentially enhance persistence. The approach offers key advantages over knockout of MHC class I, and this modification is easier to incorporate into a fully-scaled manufacturing process. Additional modifications are being explored with the goal of reducing $\mathrm{T}$ cell exhaustion and enhancing function in the suppressive tumor microenvironment.

\section{ICLEa19-0034}

Fighting Infections and Autoimmune Diseases

\section{“ONE SIZE FITS ALL" STRATEGY FOR T CELL CORRECTION, SELECTION AND DEPLETION AS NEW TREATMENT FOR HIGM1 SYNDROME}

V. Vavassori ${ }^{1}$, E. Mercuri ${ }^{1}$, G. Schiroli ${ }^{1}$, G. Marcovecchio ${ }^{1}$, M.C. Castiello ${ }^{1}$, A. Annoni ${ }^{1}$, L. Albano ${ }^{1}$, C. Margulies ${ }^{2}$, F. Buquicchio ${ }^{2}$, C. Cotta Ramusino ${ }^{2}$, A. Villa ${ }^{1}$, $\underline{\text { L. Naldini }}^{1}, \underline{\text { P. Genovese }}^{1,3}$

${ }^{1}$ San Raffaele Telethon Institute for Gene Therapy SR-Tiget, San Raffaele Scientific Institute, Milan, Italy ${ }^{2}$ Editas Medicine, Inc., Boston, USA ${ }^{3}$ Gene Therapy Program, Dana-Farber/Boston Children's Cancer and Blood Disorders Center, Boston, USA

X-linked hyper-IgM syndrome (HIGM1) is caused by mutations of $C D 40 L G$, whose absence in CD4 T-cells impairs their helper signaling for B-cell activation/immunoglobulin class-switching. Since its unregulated expression caused lymphoproliferation/lymphomas, we aimed to correct CD4OLG while preserving its physiologic regulation. Corrected autologous T-cells could provide immediate therapeutic benefit to patients by resolving pre-existing infections and bridge them towards a definitive cure by Hematopoietic-Stem/Progenitor-Cell (HSPC) transplant. To validate this strategy, we infused wild-type T-cells into HIGM1 mice pre-conditioned or not with different lymphodepleting regimens, reaching long-term, stable T-cell engraftment and partial rescue of antigen-specific IgG response upon vaccination. Thus, we optimized a CRISPR/Cas9-based protocol to insert a corrective cDNA into the first intron of CD4OLG in human T-cells and correct most disease-causing mutations with the same highly-specific reagents. This strategy allows $\sim 40 \% \mathrm{~T}$-cell correction while preserving the long-term-repopulating T-stemmemory cells. CD40L expression and physiologic regulation was restored on edited CD4+ T-cells from both healthy donors and 
HIGM1 patients, reaching up-to $60 \%$ of wild-type expression level. Corrected T-cells provided normal contact-dependent helper function to B-cell as assessed by in-vitro proliferation, classswitching and IgG secretion assays. To increase the yield of edited T-cell before transplant, we coupled the corrective cDNA with a clinical-compatible selector gene and confirmed that enriched T-cells preserved their engraftment capacity in NSG mice. Surprisingly, the presence of the selector gene in the transcript allows higher CD40L expression that was now comparable to healthy controls. Since surface expression of the reporter gene was observed also in resting cells, we exploited an optimized, truncated version of the EGFR selector gene for allowing in vivo tracking of the edited cells and, in case of adverse events, their depletion by a pharmaceutical-grade monoclonal antibody. Our work establishes the rationale and guiding principles for clinical translation of $\mathrm{T}$ cells gene correction for treating HIGM1 patients.

\section{ICLEa19-0029}

Fighting Infections and Autoimmune Diseases

\section{HIV-SPECIFIC HUMORAL IMMUNE RESPONSES BY CRISPR/CAS9-EDITED B CELLS}

H. Hartweger $^{1}$, A. McGuire ${ }^{2}$, M. Horning ${ }^{1}$, J. Taylor $\mathrm{J}^{2}$, P. Dosenovic ${ }^{1}$, D. Yost ${ }^{1}$, A. Gazumyan ${ }^{1}$, M. Seaman $S^{3}$, L. Stamatatos ${ }^{2}$, M. Jankovic ${ }^{1}$, M. Nussenzweig $C^{1}$

${ }^{1}$ The Rockefeller University, Laboratory of Molecular Immunology, New York- NY, USA ${ }^{2}$ Fred Hutchinson Cancer Research Center, Vaccine and Infectious Disease Division, Seattle, USA ${ }^{3}$ Beth Israel Deaconess Medical Center, Center for Virology and Vaccine Research, Boston, USA

A small number of HIV-1-infected individuals develop broadly neutralizing antibodies to the virus (bNAbs). These antibodies are protective against infection in animal models. However, they only emerge 1-3 yr after infection, and show a number of highly unusual features including exceedingly high levels of somatic mutations. It is therefore not surprising that elicitation of protective immunity to HIV-1 has not yet been possible. Here we show that mature, primary mouse and human B cells can be edited in vitro using CRISPR/Cas9 to express mature bNAbs from the endogenous Igh locus. Moreover, edited B cells retain the ability to participate in humoral immune responses. Immunization with cognate antigen in wild-type mouse recipients of edited B cells elicits bNAb titers that neutralize HIV-1 at levels associated with protection against infection. This approach enables humoral immune responses that may be difficult to elicit by traditional immunization.

\section{ICLEa19-0004}

New Horizons in Lymphocyte Engineering

\section{TRUCKS: THE FOURTH GENERATION OF CAR T CELLS FOR ADVANCED ADOPTIVE CELL THERAPY}

H. Abken $^{1}$

\footnotetext{
${ }^{1}$ University Regensburg, RCI-Chair Gene Immunotherapy,
} Regensburg, Germany

Adoptive therapy with chimeric antigen receptor (CAR) modified $\mathrm{T}$ cells achieved spectacular remissions of so far refractory leukemia/lymphoma, mostly in the treatment of B cell malignancies, resulting in lasting remissions. While the treatment of solid tumors also showed some efficacy, the successful treatment of solid cancer remains challenging. This is thought to be due to the hostile conditions of the tumor stroma and the repressive immune cell infiltrate. In new developments, CAR T cells are used as "living factories" to deposit immune modulating cytokines in the targeted tumor tissue aiming at converting the immune cell environment into a more favorite one to sustain a productive anti-tumor response. We designed the strategy to engineer CAR T cells with a CAR inducible expression cassette driven by the NFAT(6)-IL2 minimal promoter in order to release a heterologous protein upon CAR recognition of target. Such TRUCKs (T cells redirected for unrestricted cytokine release and killing) releasing IL-12 are shown superior in attracting and activating the innate immune response in the tumor lesion. TRUCKs releasing IL-18 displayed a Tbet(high) FoxO(low) signature with improved killing capacities. IL-18 TRUCKs moreover altered the tumor environment by increasing Th17 cells and decreasing Treg cells in the targeted tumor lesion. TRUCKs or "fourth generation" CAR T cells are going to change our concepts of treating tumors and delivering drugs to pre-defined lesions in the near future.

\section{ICLEa19-0011}

New Horizons in Lymphocyte Engineering

\section{GATED TARGETING WITH A NOVEL SWITCHABLE CAR PLATFORM TECHNOLOGY}

A. Feldmann $^{1}$, A. Hoffmann ${ }^{1}$, E. Kittel-Boselli ${ }^{2}$, R. Bergmann ${ }^{1}$,

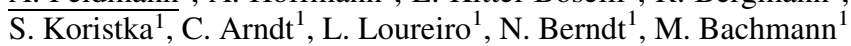

${ }^{1}$ Helmholtz-Zentrum Dresden-Rossendorf HZDR, Institute

of Radiopharmaceutical Cancer Research, Dresden, Germany

${ }^{2}$ University Hospital Carl Gustav Carus-TU Dresden,

University CancerCenter UCC, Dresden, Germany

T-cells armed with conventional CARs (cCARs) are highly effective especially in hematological malignancies. However, they often fail against solid tumors, induce tumor escape variants and cause life-threatening side effects. The safety of cCAR therapy should be improved by on/off-switchable CARs. Additionally, gated targeting strategies could increase the CAR specificity, minimize on-target/off-tumor toxicities and reduce tumor escape variants. For AND gate targeting, the signaling and costimulatory motifs are split from one onto two separate CARs of different specificities. Dual-CAR-T-cells, expressing both CARs, get activated only after recognizing both antigens. However, the application of such pairs of cCARs is very challenging as the affinity and signal strength of both CARs have to be adapted accordingly. Furthermore, the cCAR size limits the number of specificities that can be simultaneously transduced into a T-cell.

Therefore, our idea was to replace the extracellular scFv domain of cCARs with a small peptide epitope. Resulting RevCARs have a small size, avoid unspecific binding and tonic signaling caused by $\mathrm{scFv}$ dimerization. RevCAR-T-cells can be redirected against tumors only via bispecific target modules. Such RevTMs can be used as on/off-switch of RevCAR-T-cells and flexibly replaced for targeting of any antigens. For proof of concept, two RevCARs with different peptide epitopes and a series of respective RevTMs were constructed and functionally proven for targeting of leukemic as well as solid cancers. Moreover, for gated targeting, two RevCARs were expressed in the same T-cell that differ in their extracellular peptide epitope, transmembrane and intracellular signaling domains to separately transmit isolated activation and costimulatory signals. For efficient activation of Dual-RevCAR-T-cells, both RevCARs must be engaged by respective RevTMs recognizing different epitopes and antigens. 
In summary, we established a novel switchable, modular and adaptable RevCAR system characterized by small size, improved safety, easy controllability, and high flexibility allowing gated targeting strategies.

\section{ICLEa19-0010}

New Horizons in Lymphocyte Engineering

\section{UNICAR T-CELLS RETARGETED VIA SHORT-LIVED AND EXTENDED HALF-LIFE TARGET MODULES - A COMBINED APPROACH FOR CANCER IMMUNOTHERAPY}

L. Loureiro $^{1}$, A. Feldmann ${ }^{1}$, R. Bergmann ${ }^{1}$, N. Berndt ${ }^{1}$, A. Hoffmann $^{1}$, N. Mitwasi ${ }^{1}$, J. Jureczek ${ }^{2}$, S. Koristka ${ }^{1}$, M. Bachmann ${ }^{1}$, C. Arndt ${ }^{1}$

${ }^{1}$ Helmholtz-Zentrum Dresden - Rossendorf HZDR, Institute of Radiopharmaceutical Cancer Research, Dresden, Germany ${ }^{2}$ German Cancer Consortium DKTK, German Cancer Research Center DKFZ, Heidelberg, Germany

Chimeric antigen receptors (CARs) are highly efficient tools for T-cell-based cancer immunotherapy. Nonetheless, this approach is associated with mild to severe toxicities including cytokine release syndrome and on-target/off-tumor effects. To increase clinical safety while maintaining the efficacy of CAR T-cell therapy, we developed a novel modular universal CAR (UniCAR) platform. UniCAR T-cells are exclusively activated via a target module (TM) that establishes the cross-link between UniCAR T-cells and cancer cells. Given the small size of such molecules, they are rapidly eliminated and thus, have to be administrated via continuous infusion. Consequently, activation and possible side effects of UniCAR T-cells can be easily controlled by TM dosing. Regulation of CAR T-cell activity is mainly important during onset of therapy when tumor burden and the risk for severe side effects are high. Therefore, TMs with extended half-life may improve eradication of residual tumor cells in late phase of treatment and further expedite clinical application. In this line of thought, we developed both short-lived and longer lasting TMs directed against several tumor-associated antigens. Short-lived TMs are composed of a tumor-specific binding moiety and the E5B9 peptide epitope which is recognized by UniCAR T-cells. In order to generate extended half-life TMs, these two components are fused to the human IgG4 Fc domain. Both shortlived and longer lasting TMs efficiently redirect UniCAR T-cells to cancer cells in a highly target-specific manner, thereby promoting the secretion of pro-inflammatory cytokines and tumor cell lysis in vitro and in vivo. As demonstrated by PET-imaging, all TMs specifically enriched at the tumor site presenting either short or prolonged serum half-lives. Taken together, combination of different short-lived and extended half-life TMs provides a highly promising and customized tool for retargeting of UniCAR T-cells in a flexible, individualized and safe manner at different phases of tumor therapy.

\section{ICLEa19-0028}

New Horizons in Lymphocyte Engineering

\section{AVIDCARS - A NOVEL PLATFORM FOR INTEGRATION OF MULTIPLE INPUT SIGNALS BY ONE CAR MOLECULE}

$\underline{B}$ Salzer ${ }^{1}$, C.U. Brey ${ }^{1}$, C. Schüller ${ }^{1}$, M. Schöber ${ }^{1}$, E. Lobner ${ }^{2}$, ${ }_{\text {O. Dushek }}^{3}$, W. Holter ${ }^{1}$, M.W. Traxlmayr ${ }^{4}$, M. Lehner ${ }^{1}$
${ }^{1}$ Children's Cancer Research Institute, Development of Cellular Therapeutics, Vienna, Austria ${ }^{2}$ University of Natural Resources and Life Sciences, Institute of Biotechnology, Vienna, Austria ${ }^{3}$ University of Oxford, Sir William Dunn School of Pathology, Oxford, United Kingdom ${ }^{4}$ University of Natural Resources and Life Sciences, Department of Chemistry-Division of Biochemistry, Vienna, Austria

$\mathrm{T}$ cells engineered to express chimeric antigen receptors (CAR T cells) have shown high potency in the treatment of B cell-malignancies. Despite their clinical success in haematological disorders, their broader application, especially for the treatment of solid tumours, is limited by the lack of exclusive tumour antigens and poor controllability of CAR T cells in vivo.

Here, we report a novel avidity-controlled CAR (AvidCAR) platform with inducible and logic functions which improves controllability and tumour specificity of CAR T cells. Our approach is based on the combination of (i) an improved CAR design which enables controlled dimerization and (ii) a significant reduction of antigen binding affinities to introduce dependence on bivalent interaction, i.e. the exploitation of the avidity-effect resulting from amplification of individual affinities.

We prove that the activity of AvidCARs can be efficiently controlled by induced dimerization, thereby creating an ON-switch CAR. In addition, we constructed AvidCARs with AND-gate function for combinatorial recognition of either two different surface antigens or the combination of a surface antigen with a secreted tumour-associated protein. In vivo experiments in a murine leukaemia model show that AvidCARs allow for regulation of CAR $T$ cell activity by a clinically applicable drug and for the first time enable efficient combinatorial targeting of two different target antigens on the same cell.

Taken together, we present a novel CAR platform (i.e. AvidCARs), which facilitates the integration of inducible and logic control functions into CAR molecules. We anticipate that AvidCARs have the potential to be broadly used to precisely control CAR T cell activity and specificity.

\section{ICLEa19-0052}

Non-Viral Vectors and Transposons

\section{DEVELOPMENT OF CAR-T CELL THERAPY FOR B-ALL USING A POINT-OF-CARE APPROACH}

L. Abdo ${ }^{1}$, M. Viegas ${ }^{1}$, L. Marques ${ }^{1}$, P. Ferreira ${ }^{1}$, L. Barros $^{1}$, L. Chicaybam $^{2}, \underline{\text { M. Bonamino }}{ }^{1}$

${ }^{1}$ Brazilian National Cancer Institute INCA, Research Coordination, Rio de Janeiro, Brazil ${ }^{2}$ Oswaldo Cruz Foundation FIOCRUZ, Vice Presidency of Research and Biological Collections VPPCB, Rio de Janeiro, Brazil

CD19-targeted CAR-T cell immunotherapy is now an approved treatment for $\mathrm{B}$ cell leukemias and lymphomas. Current protocols for producing CAR-T cells use viral vectors for genetic modification and take 12-15 days to expand sufficient numbers of $\mathrm{T}$ cells, constituting an important bottleneck for the widespread use of this therapy. The development of non-viral, plasmid-based systems like Sleeping Beauty (SB) transposons, coupled with the emergence of large scale, closed electroporation devices, present the opportunity of generating high numbers of CAR-T cells in a short period of time and transforming this in a point-of-care (POC) therapy. In this work, mononuclear cells were isolated using Ficoll and electroporated using Nucleofector IIb combined with plasmids enconding 19BBz CAR (in the pT3 SB transposon backbone) and SB100x transposase. Cells were rested for $24 \mathrm{~h}$ and 
used for in vitro and in vivo experiments. CAR expression on day 1 following electroporation ranged between $5-15 \%$ and in vitro cytotoxic activity against RS4;11 or Nalm-6 CD19+ B cell precursor leukemia lines was low or absent. NSG mice engrafted with RS4;11 and treated with $1 \times 10^{5} 19 \mathrm{BBz}+$ cells showed improved survival when compared to mice treated with mock electroporated cells. POC approach was also efficient against Nalm-6, where $19 \mathrm{BBz}+\mathrm{CART}$ cells $\left(7 \times 10^{5}\right.$ per mice) improved survival of mice. In both models, decreased tumor burden in blood and spleen was observed in mice treated with 19BBz. Side-byside comparison of $19 \mathrm{BBz}$ cells used in POC approach or expanded for 8-12 days in vitro showed similar antitumor activity in vivo against RS4;11 cells, leading to equivalent improvements in mice survival. In summary, we showed that POC strategy is a viable approach for the use of CAR-T cells, obviating the need for $\mathrm{T}$ cell expansion while having the potential to greatly decrease the total cost and time of CAR-T cell therapy manufacturing.

\section{ICLEi19-0011}

Non-Viral Vectors and Transposons

\section{ADVANCING CELLULAR THERAPIES VIA NON-VIRAL ENGINEERING OF HEMATOPOIETIC CELLS AND STEM CELLS USING CLINICAL ELECTROPORATION}

\author{
${\underline{\text { C. } \text { Mann }^{1}}}^{1}$, J. Brady ${ }^{1}$, R. Shivakumar ${ }^{1}$, D. Acharya ${ }^{1}$ \\ ${ }^{1}$ MaxCyte-Inc, Applications, Gaithersburg, USA
}

Excitement over recent promising breakthroughs in autologous cellular therapies has been tempered by the manufacturing costs associated with viral-based gene delivery methods and by safety concerns associated with random integration of viral vectors. As an alternative to viral-based gene delivery, we describe the use of MaxCyte's clinically-validated, scalable electroporation technology for delivering mRNA, transposons, and ribonuclear proteins into primary $\mathrm{T}$ cells, natural killer cells, hematopoietic stem cells and induced pluripotent stem cells. We include data to show efficient expression of chimeric antigen receptors and $\mathrm{T}$ cell receptors via mRNA delivery and transposonmediated gene insertion into $\mathrm{T}$ cells and NK cells. We also show highly efficient gene editing following transfection of CRISPR/ Cas 9 and zinc finger nuclease reagents into T cells, NK cells and iPSCs. Finally, we highlight key attributes of the MaxCyte platform, such as reproducibility and scalability, while including data from actual case studies of cellular therapies that are being developed using the MaxCyte cell engineering platform.

\section{ICLEa19-0014}

TCR Targeting Solid Tumors

\section{TCR/CAR GENE-MODIFIED ALLOGENEIC GAMMA/DELTA-T CELLS FOR THE TREATMENT OF HEMATOLOGICAL CANCERS}

H. Fujiwara ${ }^{1}$, S. Okumura ${ }^{1}$, H. Miwa ${ }^{1}$, Y. Akahori ${ }^{1}$, Y. Miyahara $^{1}$, L. Wang ${ }^{1}$, I. Tawara ${ }^{2}$, T. Jo ${ }^{3}$, Y. Tanaka ${ }^{4}$, H. Ikeda ${ }^{5}$, H. Shiku ${ }^{1}$

${ }^{1}$ Mie University, Department of Personalized Cancer
Immunotherapy, Tsu, Japan ${ }^{2}$ Mie University Hospital,
Department of Hematology and Oncology, Tsu, Japan ${ }^{3}$ Japanese
Red Cross Nagasaki Genbaku Hospital, Department
of Hematology, Nagasaki, Japan ${ }^{4}$ Nagasaki University, Center
for Bioinformatics and Molecular Medicine, Nagasaki, Japan
${ }_{5}^{5}$ Nagasaki University, Department of Oncology, Nagasaki, Japan
Background and purpose: Clinical utility of TCR/CAR gene-modified allogeneic gamma/delta (g/d)-T cells has yet been fully characterized. In this study, we preclinically attempted to examine therapeutic potential of allogeneic g/d-T cells genemodified to express tumor antigen-specific TCR or CAR against hematological cancers.

Methods: Allogeneic Vgamma 9/delta2 (allo-Vg9/d2)-T cells from healthy donors were successfully expanded using our novel bisphosphonate derivative PTA (tetrakis-pivaloyloxymethyl2(thiazole-2-ylamino) ethylidene-1,1-bisphosphonate) and 10 daysexpansion with recombinant human (rh) IL-7 and rh IL-15, thereby subjected to retroviral TCR or CAR gene-modification. Firstly, HLA-A24-restricted and HTLV-1 Tax-specific TCRalpha/beta genes generated from adult $\mathrm{T}$ cell leukemia (ATL) patinets were retrovirally transduced, and those TCR genemodified allo- $\mathrm{Vg} 9 / \mathrm{d} 2-\mathrm{T}$ cells were subjected to functional assays against HTLV-1 infected and ATL cell lines in vitro and in vivo. Next, three diffrent CD19 CAR gene-constructs including CD28CD3 zeta (28z), CD3 zeta-Glucocorticoid-induced TNFR-related protein (GITR) (Zg) and 4-1BB-CD3 zeta (4-1BBz), respectively, were similarly introduced, and those CD19-CAR gene-modifiedallo- $\mathrm{Vg} 9 / \mathrm{d} 2-\mathrm{T}$ cells were also functionally assessed.

Results: Allo- $\mathrm{Vg} 9 / \mathrm{d} 2-\mathrm{T}$ cells were successfully expanded in a large scale with $>90 \%$ purity within 10 days. Besides TCR/CAR molecules, those allo- $\mathrm{Vg} 9 / \mathrm{d} 2-\mathrm{T}$ cells displayed natural cytotoxic receptors, which might contribute to their quick anti-tumor activities. HTLV-1 Tax-specific TCR gene-modified allo-Vg9/d2-T cells successfully killed ATL cell line cells in an HLA-A24restricted fashion both in vitro and in vivo, without causing xenogenic graft versus host disease. Those allo-Vg9/d2-T cells gene-modified with three different CAR constructs comparably produced multiple cytokines in response to NALM6 cell line cells. Real-time cytotoxicity assay (xCELLigence) demonstrated the superiority of CD19-Zg in quickness, maximal grade and duration of cytotoxicity, compared with those by the remainings.

Conclusions: Although further studies are warranted, using our novel bisphosphonate derivative and expansion culture, TCR/CAR gene-modified allo-Vg9/d2-T cells strongly suggested their therapeutic potentials against hematological cancers. Taken those, clinical trials are now being prepared.

\section{ICLEi19-0007}

\section{TCR Targeting Solid Tumors}

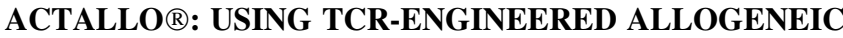 GAMMA-DELTA T CELLS TO TREAT CANCER}

M. Mata ${ }^{1}$, M. Kalra ${ }^{1}$, Z. Coughlin ${ }^{1}$, A. Nowicka ${ }^{1}$, N. Pawlowski ${ }^{2}$, F. Schwoebel ${ }^{2}$, L. Alten ${ }^{3}$, S. Kuttruff-Coqui ${ }^{2}$,

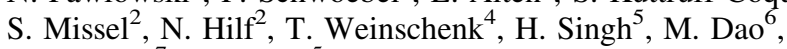
Y. Bulliard ${ }^{7}$, S. Walter ${ }^{5}$

${ }^{1}$ Immatics US Inc, Chemistry Manufacturing \& Control, Houston, USA ${ }^{2}$ Immatics Biotechnologies GmbH, Translational Development, Tuebingen, Germany ${ }^{3}$ Immatics Biotechnologies GmbH, Immunology, Tuebingen, Germany ${ }^{4}$ Immatics Biotechnologies GmbH, Discovery, Tuebingen, Germany ${ }^{5}$ Immatics US Inc, Sr Management, Houston, USA ${ }^{6}$ Immatics US Inc, Translational Development, Houston, USA ${ }^{7}$ Immatics US Inc, Translational Development Program Management, Houston, USA

Adoptive cellular therapy (ACT) has changed the landscape of cancer immunotherapy; however, the complex manufacture of individualized products from late-stage, heavily pre-treated 
cancer patients, together with high cost-of-goods, encourages the development of simpler 'off-the-shelf' alternatives. The unique properties of gd (gamma-delta) $\mathrm{T}$ cells make them an attractive candidate for effective allogeneic ACT. In contrast to ab T cells, the gd $\mathrm{T}$ counterparts are endowed with intrinsic anti-tumor activity while retaining their allogeneic nature and thus are unable to induce graft-versus-host disease. These gd T cells have a high proliferative potential before exhibiting exhaustion. Moreover, in the context of ab TCR-engineered ACT, gd T cells pose a low risk of off-target toxicity, due to the absence of expression of endogenous ab TCR and hence, no possibility of mispairing with the engineered $a b$ TCR.

Here, we present pre-clinical data from an allogeneic (ACTallo $\left.{ }^{\circledR}\right)$ platform, which combines the unique properties of $\mathrm{gd} \mathrm{T}$ cells with Immatics' XPRESIDENT ${ }^{\circledR}$ target-discovery and
XCEPTOR ${ }^{\circledR}$ TCR-discovery platforms. The ACTallo ${ }^{\circledR}$ process selectively expands $\mathrm{Vg} 9 \mathrm{Vd} 2 \mathrm{~T}$ cells from healthy donors that are transduced to co-express CD8-coreceptor together with an ab TCR targeting a tumor associated peptide antigen. ACTallo ${ }^{\circledR} \mathrm{gd}$ $T$ cells expand exponentially over a period of 21 days or less. The gd T-cell products generated using the current process exhibit central and effector memory phenotype. Further, ACTallo ${ }^{\circledR}$ gd $\mathrm{T}$ cells recognize tumor cells and elicit antitumor effector response comprising cytokine secretion and tumor-cell lysis.

ACTallo ${ }^{\circledR}$ is intended to overcome the limitation of autologous cellular therapies, by combining the large-scale expansion potential of allogeneic gd $\mathrm{T}$ cells with highly specific TCRs against novel tumor targets identified and characterized in-depth by the mass spectrometry-guided XPRESIDENT ${ }^{\circledR}$ technology.

\section{ICLE 2019-E-Poster Presentations}

\section{ICLEa19-0021}

CARs Targeting Solid Tumors

\section{DEVELOPMENT OF TCR-LIKE SCFV ZG CAR THAT SPECIFICALLY RECOGNIZES MAGE-A4230-239/HLA-A*02:01 COMPLEX}

Y. Akahori $^{1}$, Y. Kato ${ }^{2}$, Y. Miyahara ${ }^{3}$, C. Amaike $^{3}$, L. Wang ${ }^{3}$, K. Takuma ${ }^{4}$, H. Fujiwara ${ }^{3}$, H. Miwa ${ }^{5}$, H. Ikeda ${ }^{6}$, H. Shiku ${ }^{3}$

${ }^{1}$ Mie University, Department of Immuno-Gene Therapy, Tsu city, Japan ${ }^{2}$ Mie Univercity, Department of Immuno-Gene Therapy, Tsu, Japan ${ }^{3}$ Mie University, Department of Immuno-Gene Therapy, Tsu, Japan ${ }^{4}$ Mie University, Department of Immunology, Tsu, Japan ${ }^{5}$ Mie University, Immuno-Gene Therapy, Tsu, Japan ${ }^{6}$ Nagasaki University, Department of Oncology, Nagasaki, Japan

Aims: We aimed to develop CAR therapy utilizing TCR-like antibody that can recognize tumor specific intracellular antigenderived peptide in association with MHC class I molecule. In this report, we show our success in isolating antibody clone \#17 which specifically recognizes the HLA-A*02:01 complexed with a cancer-testis antigen MAGE-A4 $4_{230-239}$ peptide but not other peptides including those derived from the MAGE family members with homology to MAGE-A4. Utilizing the scFv derived from \#17 antibody, we constructed a CAR and investigated its specific tumor cell recognition and in vivo tumor suppressing property.

Materials and methods, Results: By screening a phage display library of human antibody with recombinant HLAA*02:01 complexed with MAGE-A4 $230-239$, we selected \#17 and constructed CAR with CD28 and CD3 zeta (28z form) and CD3 zeta and GITR (zG form) for intracellular signal transduction domain. By transducing PBMC with retrovirus vector, we produced CAR T cells that specifically recognized and lysed MAGEA4 peptide pulsed T2 cells, but not recognize T2 cells pulsed with other off-target candidate peptides including MAGE family ones.

\#17 CAR T cells specifically recognized and lysed HLAA*02:01+ MAGE-A4+ cells but not HLA- A*02:01- nor MAGE-A4- cells,
In vivo tumor model analysis was performed by inoculating NW-MEL38 cells (HLA- A*02:01+ MAGE-A4+) into immunodeficient NOG mice and infusing human CAR-T cells with $28 \mathrm{z}$ and $\mathrm{zG}$ forms. Specific tumor suppression associated with proliferation and tumor infiltration of infused CD8+ T cells were found especially in $\mathrm{zG}$ form CAR.

Conclusion: \#17 CAR was shown to specifically recognize and lyse tumor cells that endogenously express MAGE-A4 $4_{230-239} /$ HLA-A*02:01 complex. Adoptive transfer of \#17 CAR into tumor-bearing NOG mice specifically suppressed the tumor cells that express the cognate intracellular target antigen. These results suggest that the \#17 zG CAR therapy will be a promising therapy of cancer patients.

\section{ICLEa19-0033}

CARs Targeting Solid Tumors

\section{TARGETING MYELOID-DERIVED SUPPRESSOR CELLS AND IN SITU VACCINATION TO ENHANCE EFFICACY OF CAR T CELLS IN SOLID TUMORS}

E. Conde-Gallastegi ${ }^{1}$, J. Suarez ${ }^{1}$, E. Elizalde ${ }^{1}$, E. Vercher ${ }^{1}$, U. Mancheño $^{1}$, N. Casares ${ }^{1}$, T. Lozano ${ }^{1}$, J.J. Lasarte ${ }^{1}$, S. Hervás-Stubbs ${ }^{1}$

${ }^{1}$ CIMA Universidad de Navarra, Immunology and immunotherapy program, Pamplona, Spain

Chimeric antigen receptor (CAR) $\mathrm{T}$ cell therapy represents a revolutionary treatment for $\mathrm{B}$ cell malignancies. However, attempts to recapitulate this success in solid tumors have been disappointing. Among the hurdles encountered for the application of CAR T cells to solid tumors are the immunosuppressive effect of tumor microenvironment and the propagation of tumor via target antigennegative cells. To address this question, we have developed a CAR against human prostate-specific membrane antigen (hPSMA) and a syngeneic and immunocompetent mouse model expressing hPSMA. Adoptive transfer of anti-hPSMA CAR T cells in this model yielded a modest antitumor efficacy. We have observed that 
treatment with CAR T cells increased the number of granulocytic myeloid-derived suppressor cells (gMDSC) in the tumor microenvironment, indicating that tumors can amplify this population as a strategy to evade CAR T cell attack. Tumor escape was also associated with a loss of hPSMA expression by tumor cells and a notably decreased in the number of tumor-infiltrating effector cells, such as CD8, CD4, NK and NKT lymphocytes. We have demonstrated that the combination of CAR T cell therapy with strategies targeting g-MDSC synergistically triggered tumor regression. On the other hand, intratumoral administration of immunomodulators enhanced the efficacy of CAR T cell therapy by promoting the antigen spreading. Our data provide a rational for the combination of either therapies targeting MDSC or in situ vaccination to enhance the efficacy of CAR T cells in solid tumor.

\section{ICLEa19-0027}

CARs Targeting Solid Tumors

\section{SUPPRESSION OF T CELLS THROUGH IN VITRO POLARISED MYELOID CELLS AS A BASIS FOR HIGH THROUGHPUT DRUG SCREENINGS TO IMPROVE IMMUNOTHERAPY OF SOLID CANCERS}

\author{
$\underline{\text { J. Frosch }}^{1}$, L. Gargiulo ${ }^{1}$, C. Hanif Muflihah ${ }^{1}$, J. Anderson ${ }^{1}$ \\ ${ }^{1}$ UCL Institute of Child Health, Developmental Biology \\ and Cancer, London, United Kingdom
}

Neuroblastoma is a paediatric extracranial solid tumour with poor survival rates among high risk patients; despite multimodal approaches, clinical outcomes remain poor. Chimeric antigen receptor (CAR) $\mathrm{T}$ cells have been proven to be an effective cell-based treatment in leukaemia but have yet to be established in solid tumours. The genetically engineered $\mathrm{T}$ cells are re-directed to detect highly-expressed surface antigens of the tumour, leading to an intracellular activation of effector functions. However, the immunosuppressive environment formed by solid tumours heavily affects the efficacy of CAR T cell therapy due to inhibition of proliferation and effector function. Myeloid derived suppressor cells (MDSC) are a key group among local tumour-induced immune cells and have been shown to positively correlate with cancer stage, disease progression, and resistance to standard therapies in solid cancers.

In an in vitro neuroblastoma-based conditioning culture, healthy monocytes were polarised to mimic MDSC phenotype and suppressive functions. Generated neuroblastoma-conditioned monocytes mainly reflected the phenotype of highly suppressive monocytic MDSC (CD14+ HLA-DR ${ }^{\text {low }}$ ). In a co-culture-based assay, these cells were used to study effects on $\mathrm{T}$ cell proliferation and effector functions. Polarised monocytes were able to significantly inhibit proliferation of unmodified T cells as well as CAR-transduced $\mathrm{T}$ cells. Furthermore, supernatants of co-cultures containing conditioned monocytes showed significantly lower interferon gamma levels, compared to controls lacking suppressor cells. Lastly, we showed that sunitinib malate, a tyrosine kinase inhibitor, can partially recover $\mathrm{T}$ cell proliferation through inhibition of myeloid cells.

Our findings suggest that in vitro generated suppressive monocytes provide a valuable component to study effects on $\mathrm{T}$ cells in the tumour microenvironment which can be used in high throughput drug screening assays for MDSC-inhibitory drugs. This could reveal suitable candidate drugs for combination therapies used alongside CAR T cells to improve the efficacy of cell-based therapy in solid tumours.

\section{ICLEa19-0020}

CARs Targeting Solid Tumors

\section{CAR-BASED IMMUNOTHERAPY OF EGFR-POSITIVE SOLID TUMOURS USING CYTOKINE-ACTIVATED PERIPHERAL BLOOD KILLER CELLS}

\author{
$\underline{\text { O. Gavriliuc }}^{1,2}$, R. Zogorean ${ }^{1}$, F. Bojin ${ }^{1,2}$, V. Paunescu ${ }^{1,2}$ \\ I,'Pius Brinzeu" Clinical Emergency County Hospital \\ Timisoara, OncoGen Center for Gene and Cellular Therapies \\ in the Treatment of Cancer, Timisoara, Romania \\ 2 ,'Victor Babes" University of Medicine and Pharmacy \\ Timisoara, Immunology, Timisoara, Romania
}

Overexpression of EGFR, a receptor tyrosine kinase member of the ErbB family, has been observed in several kinds of human malignancies such as non-small-cell lung cancer, breast cancer, prostate cancer, gliomas, colorectal cancer or squamous cell carcinomas of the head and neck and is often associated with tumour recurrence, neoangiogenesis and metastasis. Consequently, the development of an EGFR-targeted chimeric antigen receptor therapy might prove beneficial for many cancer patients if some of the challenges associated with the translation of CAR$\mathrm{T}$ cell therapy to solid tumours are overcome.

In our study, we used a mixed population of NK-polarized activated peripheral blood-derived killer cells transduced with a cetuximab-based chimeric antigen receptor as cytotoxic effector cells capable of lysing EGFR+ tumour cells. The effector cell population was generated by IL-2/IL-15/IL-21 combined cytokine stimulation of PBMCs resulting in $>70 \%$ mixed phenotype NKT (CD3+CD56+) and NK (CD3-CD56+) cells, starting with day 10 of cell culture. Lentiviral transduction of the EGFR-CAR vector into activated cells did not affect the phenotypic conversion, with most of the CAR-expressing cells being effector memory CD56+ cells (NKem), with potent MHC-unrestricted cytotoxicity. EGFR-CARNKem cells demonstrated significantly higher cytolytic activity against EGFR positive solid tumour cell lines compared to EGFRCAR-NK92 and EGFR-CAR-T cells, while also retaining NK cell receptor mediated recognition and killing of EGFR negative cells.

\section{ICLEa19-0038}

CARs Targeting Solid Tumors

\section{POTENTIAL CANCER STEM CELL TARGETING CHIMERIC ANTIGEN RECEPTOR GENERATION WITH ANTIBODY VARIABLE REGION GENES IDENTIFIED BY IMMUNOGLOBULIN PROFILE ANALYSIS IN PATIENTS WITH MALIGNANT MELANOMA}

$\underline{\text { B. Kotlan }}^{1}$, S. Horvath ${ }^{2}$, K. Eles ${ }^{2}$, K. Czirbesz ${ }^{3}$, G. Naszados ${ }^{4}$, J. Olasz ${ }^{5}$, E. Farkas ${ }^{6}$, M. Ujhelyi ${ }^{6}$, A. Szollar ${ }^{6}$, A. Savolt ${ }^{6}$, O. Csuka ${ }^{5}$, M. Godeny ${ }^{4}$, M. Kasler ${ }^{7}$, G. Liszkay ${ }^{3}$

${ }^{1}$ National Institute of Oncology, Molecular Immunology and Toxicology, Budapest, Hungary ${ }^{2}$ National Institute of Oncology, Center of Surgical and Molecular Pathology, Budapest, Hungary ${ }^{3}$ National Institute of Oncology, Department of Oncodermatology, Budapest, Hungary ${ }^{4}$ National Institute of Oncology, Center of Image Analysis and Radiological Diagnostics, Budapest, Hungary ${ }^{5}$ National Institute of Oncology, Department of Pathogenetics, Budapest, Hungary ${ }^{6}$ National Institute of Oncology, Center of Oncosurgery, Budapest, Hungary ${ }^{7}$ Ministry of Human Capacities, n, Budapest, Hungary 
Background: Cancer metastasis is a major concern in patient outcome, so targeting and elimination of cancer stem cells (CSC) has become a number one issue in cancer therapeutics. Therefore, reliable and well tolerated tumor-associated antigen specific antibodies are needed.

Methods: A comprehensive antibody profile analysis was developed in our institute involving patients with metastatic melanoma. Comparative immunoglobulin variable region gene DNA sequence analyses, single chain $\mathrm{Fv}(\mathrm{scFv})$ antibody phage enzyme-linked immunosorbence assay (ELISA) and immunohistochemistry on primary cancerous cell cultures and tissue sections to detect disialylated glycosphingolipids were performed. Selected $\mathrm{scFv}$ with this unique tumor-associated binding specificity became the subject of a fully human chimeric antigen receptor (CAR) construct by Sleeping Beauty technique.

Results: Immunoglobulin variable region gene usage in the VH3 family represented the VH3-23 and VH3-21 overexpression and a cancer stem cell-associated CD34 and GD3 disialylated glycosphingolipid-targeting pattern. A fully human GD3 CAR construct could be developed.

Conclusions: Our results open up a strategy to reveal and eliminate cancerous cells with stem cell features, responsible for metastases. Genetic engineering of melanoma patient derived immune $\mathrm{T}$ cells are on the way to measure their antitumor effectivity. The present data foster CAR-T cell development for solid tumors.

Acknowledgements: The Harry J. Lloyd Charitable Trust Melanoma Research Award (2010), OTKA T048933, Fulbright No1206103, No1214104 Grants given to B. Kotlan are acknowledged. We thank Prof Dr LJN Cooper, Dr. A Korngold, Dr. T. J Laskowski, Dr. B. Jena, Dr N Belousova at the MDACC and Prof Dr M. Glassy (Nascent Biologics Inc.) and Prof. M. H. Ravindranath (Terasaki Foundation Laboratory) for their substantial help.

\section{ICLEa19-0049}

CARs Targeting Solid Tumors

\section{OPTIMIZING CRISPR-CAS9 MEDIATED KNOCK-IN STRATEGY TO CREATE AN INDUCIBLE GENE EXPRESSION SYSTEM IN PRIMARY HUMAN T CELLS}

\section{Z. Odé $^{1}$, C.T. Petersen ${ }^{1}$, G. Krenciute ${ }^{1}$}

${ }^{1}$ St. Jude Children's Research Hospital, Bone Marrow

Transplantation and Cellular Therapy, Memphis, USA

T cells engineered with chimeric antigen receptors (CARs) show great promise in the treatment of some cancers. Modifying $\mathrm{T}$ cells to express CARs relies on $\mathrm{T}$ cell transduction using viral particles carrying a transgene, resulting in random DNA integration within the genome of the T cell. While this approach has proven successful and is used in generating FDA-approved CD19-CAR T cells, it is possible the transgene could integrate into a genomic locus that would lead to malignant transformation of the engineered $\mathrm{T}$ cells. In addition, manufacturing viral vectors is time consuming and expensive. One way to overcome these challenges is to use a method that relies on site-specific gene integration, in this case CRISPR-Cas9 with non-viral DNA as a template for homologydirected repair (HDR) mediated knock-in. This method provides a rapid, highly-specific and inexpensive way to engineer $\mathrm{T}$ cells. Here, we describe an optimized protocol for the site-specific knockin of a large transgene in primary human $\mathrm{T}$ cells using non-viral double stranded DNA as a repair template. We targeted the T cell receptor $\alpha$ constant (TRAC) locus for gene insertion and designed a two-gene (IL15 and GFP) insert separated by a $2 \mathrm{~A}$ sequence and homology arms complementary to the TRAC locus. To optimize the knock-in conditions we tested homology arm length, amount of template DNA, cell number and electroporation conditions. Expression of the two genes was evaluated by FACS (GFP) and ELISA (IL15). Lastly, we then applied the optimized method for site-specific transgene integration into a different locus driven by a $\mathrm{T}$ cell activation dependent promoter. We showed that the transgenes are expressed in an activation dependent manner, creating an inducible system under a natural promoter. In conclusion, this CRISPR-Cas9 based knock-in approach will open new avenues for specific, rapid and inexpensive $\mathrm{T}$ cell engineering.

\section{ICLEa19-0050}

\section{CARs Targeting Solid Tumors}

\section{IMPROVING CEA-TARGETED CAR-T CELL THERAPY FOR SOLID TUMOURS}

\author{
W. Zheng $^{1}$, G. Kueberuwa ${ }^{1}$, E. Cheadle ${ }^{1}$, A. Armstrong ${ }^{2}$ \\ ${ }^{1}$ University of Manchester, Divison of Cancer Sciences, Manchester, \\ United Kingdom ${ }^{2}$ The Christie NHS Foundation Trust, \\ Department of Medical Oncology, Manchester, United Kingdom
}

Keywords: TRUCKs, syngeneic mouse model, CEA, IL-12, TGF- $\beta$, PD-1

Whilst CD19-specific CAR-T cell therapy has shown outstanding results in the treatment of haematological malignancies, the use of CAR-T cell therapy for solid tumours has largely unsuccessful to date. Apart from the paucity of tumour-specific target antigens, preclinical studies suggest that this is partly due to several immunosuppressive mechanisms in tumours, which can hinder CAR-T cell persistence and effector functions.

The aim of the project is, therefore, to improve CAR-T cell therapy for solid tumours. To achieve this, we utilised CAR T-cells targeting carcinoembryonic antigen (CEA) and human CEA (hCEA) transgenic mouse models to examine the factors that inhibit CAR-T cell efficacy activity in solid tumours. Co-stimulation, IL-12 TRUCKs ( $T$ cells redirected for universal cytokine killing) and ssCARs ( $\mathrm{scFv}$ secreting CARs) were each evaluated for their ability to enhance anti-tumour activity of CAR T-cells.

Current results have shown that incorporation of mouse CD28 co-stimulatory domain achieves a greater $\mathrm{T}$ cell activation and improves subsequent anti-tumour activity in vitro. The secretion of IL12 from within CEA-specific CAR-T cells also enhances their ability to kill tumour cells. To overcome the immunosuppressive tumour microenvironment, CEA-specific CAR-T cells were further modified to secret TGF- $\beta$ or PD- 1 blocking scFv antibodies. Preliminary data indicates that modification to secret antibody fragments is able to confer resistance of CAR T-cells to immune suppression in vitro. Work is on-going to evaluate the anti-tumour activity of CAR-T cells in vivo and their infiltration in tumour and tissues using subcutaneous tumour model.

\section{ICLEa19-0005}

Fighting Infections and Autoimmune Diseases

\section{ENGINEERING CELL-DERIVED NANOSCALE EXOSOMES FOR ANTI-INFLAMMATION AND IMMUNOTHERAPY}

\author{
D. Levy ${ }^{1}$, M. Do ${ }^{1}$, A. Brown ${ }^{1}$, B. Lu ${ }^{1}$ \\ ${ }^{1}$ Santa Clara University, Department of Bioengineering, \\ Santa Clara, USA
}

Exosomes are cell-derived nanovesicles that mediate intercellular transfer of bioactive materials packaged inside their lumen or displayed on their surface. Due to their biocompatibility, high 
tissue-penetration and ability to shuttle a large amount of therapeutics, exosomes hold a great promise for drug delivery and therapy. By packaging therapeutic cargo inside their lumen, exosomes have been successfully used to deliver nucleic acid drugs to tissues such as the brain and deep-seeded tumors. However, displaying therapeutic proteins on their outer surface has been hypothesized but not yet attempted. As a proof of concept, we investigate (i) whether therapeutic proteins can be displayed on the outer surface of exosomes in living cells; (ii) whether such engineered exosomes can function like nanosponges to absorb inflammatory/immune-modulatory cytokines such as TNF $\alpha$ and PDL1, thus preventing them from causing inflammation and immune repression. Here we describe how we genetically express chimeric receptors on the surface of exosomes in living human cells. Furthermore, we show that engineered exosomes can be successfully prepared from conditioned media by a procedure composed of ultra-filtration and chemical precipitation. Using immune-quantification, nanoparticle tracking analysis, and confocal microscopy, we confirm that these engineered exosomes retain the normal production rate, particle size and distribution, as well as molecular markers as those of unmodified exosomes. Finally, we demonstrate that these engineered exosomes could antagonize activities of pathological cytokines in human disease models. Together our study demonstrates that engineered exosomes can serve as a novel biologic for the potential treatment of human diseases such as inflammation, autoimmunity and cancer.

\section{ICLEa19-0019}

Fighting Infections and Autoimmune Diseases

\section{DEVELOPING A CRISPR-CAS9 TOOL TO INACTIVATE THE GLUCOCORTICOID RECEPTOR AND BROADEN THE APPLICABILITY OF ANTIGEN-SPECIFIC T-CELL IMMUNOTHERAPY}

M. Alvanou ${ }^{1}$, C. Pantazi $^{2}$, K. Koukoulias ${ }^{2}$, N. Psatha ${ }^{3}$, G. Tsiolas $^{4}$, I. Vallianou ${ }^{5}$, M. Yiangou ${ }^{6}$, A. Spyridonidis ${ }^{7}$, K. Stamatopoulos ${ }^{4}$, A. Anagnostopoulos ${ }^{5}$, A. Makris ${ }^{4}$, E. Yannaki ${ }^{5}$, A. Papadopoulou ${ }^{5}$

${ }^{1}$ G.Papanikolaou Hospital/University General Hospital of Patras, Gene and Cell Therapy Unit-Haematology Department, Thessaloniki, Greece ${ }^{2}$ G.Papanikolaou Hospital/Aristotle University of Thessaloniki, Gene and Cell Therapy Unit-Haematology Department/Department of Genetics- Development and Molecular Biology, Thessaloniki, Greece ${ }^{3}$ Altius Institute for Biomedical Sciences, Altius Institute for Biomedical Sciences, Seattle, USA ${ }^{4}$ Institute of Applied Biosciences INAB, Centre for Research and Technology Hellas CERTH, Thessaloniki, Greece ${ }^{5}$ G.Papanikolaou Hospital, Gene and Cell Therapy Unit-Haematology Department, Thessaloniki, Greece ${ }^{6}$ Aristotle University of Thessaloniki, Department of Genetics- Development and Molecular Biology, Thessaloniki, Greece ${ }^{7}$ University General Hospital of Patras, Department of Haematology, Patras, Greece

Infections are fatal complications after hematopoietic stem cell transplantation (HSCT). While pharmacotherapy often fails, adoptive immunotherapy (AI) with pathogen-specific $\mathrm{T}$ cells (pSTs) is a promising alternative. However, T cells perform suboptimally under steroids, the first-line treatment of transplant-associated complications, depriving the most susceptible to infections patients of the potential benefits of AI. We here evaluated the impact of dexamethasone (DEX) on T cells and developed a CRISPR/Cas9 system to genetically disrupt the glucocorticoid receptor (GR) and confer resistance to steroids, ultimately aiming to generate steroid-resistant pSTs. A 3-day DEX exposure impaired the proliferation of CD3/CD80-pulsed primary $\mathrm{T}$ cells and induced apoptosis and transition from a central to an effector memory phenotype, while upregulated PD-1 and CTLA-4 over the "no DEX" condition. To inactivate the GR gene, T2 cells transduced with lentiviral vectors encoding Cas9 and 10 different gRNAs (targeting GR at a transcription start site or exons 2-5) in pooled libraries or separately, were incubated \pm DEX. Cells edited with 2 gRNA pools, presented normal proliferation and reduced apoptosis on DEX treatment versus their untreated counterparts, suggesting that pools contained functional gRNAs. To clarify whether DEX-resistance was a single gRNA effect or an additive/synergistic action of the pooled gRNAs, T2 cells were transduced by single gRNA vectors. In DEX's presence, cells edited with 8/10 tested gRNAs presented similar apoptosis and proliferation to the untreated cells suggesting functional resistance to DEX. GR disruption was confirmed by western blotting. Among these gRNAs, an optimal gRNA will be selected based on the highest GR disruption efficiency and the lowest off-target activity. Overall, we provide a series of gRNAs to disrupt the GR and confer resistance of $\mathrm{T}$ cells to steroids, endeavoring to generate steroid-resistant pSTs and offer the benefits of AI to the most vulnerable to infections patients as those receiving steroids post HSCT.

ICLEa19-0031

Fighting Infections and Autoimmune Diseases

\section{DEEP-PRIMED IL-15 SUPERAGONIST IMPROVES ANTIVIRAL EFFICACY OF HIV-SPECIFIC CD8+ T-CELLS IN A HUMANIZED MOUSE MODEL}

C. Mccann ${ }^{1,2}$, E. Zale ${ }^{2}$, A. Ward ${ }^{3,4}$, T. Dilling ${ }^{1,5}$,

A. Danesh $^{1}$, E. Stevenson ${ }^{1}$, T. Mota ${ }^{1}$, T. Andresen ${ }^{6}$, D. Jones ${ }^{6}$, D. Irvine ${ }^{7}$, R.B. Jones ${ }^{1,2,3}$

${ }^{1}$ Weill Cornell Medical College, Medicine, New York NY, USA

${ }^{2}$ Weill Cornell Graduate School of Medical Sciences, Immunology and Microbial Pathogenesis, New York NY, USA

${ }^{3}$ The George Washington University, Microbiology Immunology \& Tropical Medicine, Washington DC, USA ${ }^{4}$ The George

Washington University Milken Institute School of Public Health, Epidemiology, Washington DC, USA ${ }^{5}$ The Graduate Center - City University of New York, PhD Program in Chemistry, New York NY, USA ${ }^{6}$ Torque Therapeutics, Bioengineering, Cambridge MA, USA ${ }^{7}$ Massachusetts Institute of Technology, Koch Institute for Integrative Cancer Research, Cambridge MA, USA

HIV-specific $\mathrm{CD}^{+} \mathrm{T}$-cells play a critical role in partially controlling viral replication in infected-individuals, but ultimately fail to eliminate infection. Enhancing these T-cell responses through lymphocyte engineering approaches has the potential as a novel therapy capable of achieving durable control or eradication of infection.

IL-15 Superagonist (IL-15SA) potently supports the in vivo persistence and antiviral activity of adoptively transferred $\mathrm{CD} 8^{+}$ T-cells. The Deep-Priming ${ }^{\mathrm{TM}}$ technology platform, developed by Torque, allows for loading of immunomodulators onto the surface of T-cells via electrostatic 'nanogels', which slowly release to deliver sustained autocrine immune stimulation without the harmful effects of systemic exposure. Here, we investigate the impact of IL-15SA Deep-Priming on HIV-specific $\mathrm{CD}^{+} \mathrm{T}$-cells in a humanized mouse model of HIV infection. 
Humanized mice were generated by engrafting NOD-scidIL2 $\mathrm{Rg}^{\text {null }}$ mice with memory $\mathrm{CD}^{+} \mathrm{T}$-cells isolated from an ARV-suppressed HIV+ donor. An autologous HIV-specific Cytotoxic T-Lymphocyte (CTL) clone was isolated, and killing potential confirmed. Four weeks post humanization, mice were infected with HIV and received an infusion of unmodified HIVSpecific CTLs, or IL-15SA Deep-Primed HIV-specific CTLs (CTL-DP). T-cell numbers and plasma viral loads were quantified weekly by flow cytometry and qRT-PCR.

Mice receiving unmodified CTLs trended toward reduced viral loads compared to the No Treatment condition, while mice receiving CTL-DP saw significant, $2-\log _{10}$ reduction in VL $(\mathrm{p}<0.01)$. At 41 days post-infection $100 \%(5 / 5)$ of the No Treatment, $66.7 \%(4 / 6)$ of the CTL treatment, and $16.7 \%(1 / 6)$ of CTL-DP treatment mice had detectable viremia. IL-15SA Deep-Priming increased CTL expansion and persistence in peripheral blood which correlated with improved $\mathrm{CD}^{+}{ }^{-} \mathrm{T}$-cell preservation.

Here we demonstrate the first in vivo analysis of IL-15SA Deep-Priming of HIV-Specific CTLs. These data suggest that Deep-Priming of patient T-cells can enhance in vivo function and persistence, leading to improved viral suppression - a significant advancement in the field of HIV cure research.

ICLEa19-0043

Fighting Infections and Autoimmune Diseases

CHARACTERIZATION OF MHC CLASS IIRESTRICTED T-CELL RECEPTORS FOR T-CELL THERAPY OF HBV INFECTION

S. Schreiber ${ }^{1}$, M. Honz ${ }^{1}$, M. Schiemann ${ }^{2}$, A. Sette ${ }^{3}$, C. Zielinski ${ }^{1}$, U. Protzer ${ }^{1}$, K. Wisskirchen ${ }^{1}$

${ }^{1}$ Technical University of Munich/Helmholtz, Zentrum Munich, Institute of Virology, Munich, Germany ${ }^{2}$ Technical University of Munich, Institute for Medical Microbiology- Immunology and Hygiene, Munich, Germany ${ }^{3}$ La Jolla Institute

for Immunology, Center for Infectious Disease, La Jolla, USA

Hepatitis B virus (HBV) infection remains a severe health problem with current treatment options being unable to achieve viral clearance. Since virus eradication is accompanied by a strong T-cell response in patients with resolved infection, adoptive $\mathrm{T}$-cell therapy represents a promising therapeutic approach. We recently demonstrated that CD8+ $\mathrm{T}$ cells grafted with T-cell receptors (TCR) restricted by MHC class I have the potential to cure HBV infection in vitro and in vivo. Nevertheless, also CD4+ T cells are known to play an important role in resolving $\mathrm{HBV}$ infection and may therefore potentially benefit T-cell therapy. We thus identified, cloned and characterized MHC class II-restricted HBV-specific TCRs from donors with cleared HBV infection. A total of 23 TCRs specific for eight different epitopes from $\mathrm{HBV}$ envelope, core or polymerase protein were generated. All TCRs were expressed on primary T cells through retroviral transduction with transduction rates ranging from $65-85 \%$. TCR genome integration was limited to under five integrates per cell, as determined by qPCR. Seven different MHC class II restrictions were identified by co-culture with single HLA-transfected target cells as well as partially matched lymphoblastoid cell lines. 17 TCRs recognized at least three HBV genotype variants and 12 TCRs had a high binding affinity with EC50 values in a low nanomolar range. 20 TCRs were capable of activating CD8+ $\mathrm{T}$ cells, inducing strong granzyme B secretion. Taken together, our set of $23 \mathrm{MHC}$ class II-restricted TCRs recognizes epitopes derived from HBV core, surface and polymerase protein. These TCRs enable both CD4+ and CD8+ T cells to detect antigen on MHC class II in a nanomolar range, resulting in antigen-specific $\mathrm{T}$-cell activation.

\section{ICLEa19-0046}

Fighting Infections and Autoimmune Diseases

\section{VACCINE ELICITATION OF HIV BROADLY NEUTRALIZING ANTIBODIES FROM ENGINEERED B CELLS}

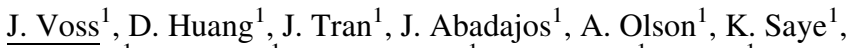
T. Blane ${ }^{1}$, R. Fuller ${ }^{1}$, L. Couvrette ${ }^{1}$, M. Tenuta ${ }^{1}$, W. Li ${ }^{1}$, T. Schiffner ${ }^{1}$, E. Landais ${ }^{2}$, A. Gonzalez-Martin ${ }^{3}$, D. Sok ${ }^{2}$, D. Burton ${ }^{1}$, W. Schief ${ }^{1}$, D. Nemazee ${ }^{1}$

${ }^{1}$ Scripps Research, Immunology and Microbiology, La Jolla, USA ${ }^{2}$ Scripps Research, IAVI Neutralizing Antibody Center, La Jolla, USA ${ }^{3}$ Universidad Autonoma de Madrid, Department of Biochemistry, Madrid, Spain

Viruses such as HIV have evolved features to avoid neutralization by most antibodies available in the human repertoire, hindering the development of universally effective vaccines. Only rarely in the context of infection have human HIV broadly neutralizing antibodies (bnAbs) been generated through the extensive affinity maturation of rare precursor $\mathrm{B}$ cells. Such bnAb genes can be added directly to the repertoire as functional antigen receptors in primary B lymphocytes using genome editing technologies. Here we show that these engineered B cells can enter germinal centers upon vaccination and mature into memory which can be boosted to generate high titer bnAb responses in the serum of wild type mice. Bypassing genetic limitations imposed by the antibody repertoire could thus allow for the development of a new generation of engineered $\mathrm{B}$ cell vaccines, capable of eliciting broadly protective immunity against HIV, Influenza, Hepatitis C, and other evolved pathogens.

\section{ICLEi19-0004}

Genome Editing for Allogeneic ACT

\section{THE TARGETED KNOCKDOWN OF CD3 $\zeta$ BY SHRNA AS A PLATFORM TO DEVELOP ALLOGENEIC CAR T CELL THERAPY}

S. Raitano ${ }^{1}$, A. Michaux ${ }^{2}$, S. Bornschein ${ }^{2}$, J. Bolsée $^{3}$, S. Lenger ${ }^{4}, \overline{\text { H. Machado }}{ }^{5}$, P. Sotiropoulou ${ }^{2}$, D. Gilham ${ }^{2}$

${ }^{1}$ Celyad, R\&D, Mont-Saint-Guibert, Belgium ${ }^{2}$ Celyad, Research and Development, Mont Saint Guibert, Belgium ${ }^{3}$ Celyad, Research and Development, Mont Saint Guibrt, Belgium

${ }^{4}$ Horizon discovery, Research and development, Lafayette, USA

${ }^{5}$ Horizon Discovery, Research and Develompment, Lafayette, USA

Allogeneic CAR-T cell therapy seeks to overcome some of the hurdles faced by the autologous approach through a more consistent starting product, bulk manufacture and banking to facilitate a time-saving 'off the shelf' therapy delivery. A key condition for the success of Allogeneic CAR-T cell therapy relies on eliminating the activity of the endogenous $\mathrm{T}$ cell receptor (TCR), essential to avoid the allogeneic CAR-T cells targeting healthy patient tissue resulting in life-threatening graft versus host disease (GvHD).

In this study, the potency of shRNA to specifically knockdown $\mathrm{CD} 3 \varepsilon$ and $\mathrm{CD} 3 \zeta$ to down-regulate TCR expression in primary $\mathrm{T}$ cells was investigated. $\mathrm{CD} 3 \varepsilon-$ and $\mathrm{CD} 3 \zeta$-targeting 
shRNA reduced the transcriptional expression of their respective targets driving a reduction in TCR cell surface expression and subsequent diminished response to mitogenic activation. The CD3 $\zeta$ shRNA showed a greater capability to knock-down TCR. This was further investigated through a comparison against $T$ cells where $\mathrm{CD} 3 \zeta$ expression was knocked-out through CRISPRCas9 gene-editing. Flow cytometric analysis showed that the cell surface TCR expression of shRNA $\mathrm{T}$ cells was reduced to a similar magnitude as $\mathrm{T}$ cells with the $\mathrm{CD} 3 \zeta$ knockout. While the control, non-modified donor cells readily drove GvHD after infusion in the NSG mouse model, there was little evidence of GvHD in either the CD3 $\zeta$-shRNA or CD3 $\zeta$-CRISPR-Cas9 T cell arms during the length of the experiment. Interestingly, while both $\mathrm{T}$ cell populations engrafted to an initially equal frequency in the periphery, the gene-edited cell population disappeared by day 54 while the shRNA cells could be readily detected throughout the experiment.

These studies suggest that targeting of CD3 $\zeta$ with shRNA reduces TCR expression to a level that controls GvHD in the NSG mouse model and provides a platform upon which to develop an allogeneic CAR T cell therapy strategy.

The study was approved by authorities and Ethics Board.

\section{ICLEa19-0044 \\ Hematological Malignancies \\ CRISPR-CAS9 MEDIATED GENERATION OF CORD BLOOD TCRAB-/- CD8+ T CELLS EXPRESSING RECOMBINANT WT1-TCR TO DECREASE RELAPSE IN ACUTE MYELOID LEUKEMIA PEDIATRIC PATIENTS}

V. Lo Presti ${ }^{1}$, E. Dunnebach ${ }^{1}$, M. Plantinga ${ }^{1}$, K. Jurgen ${ }^{1}$, S. Nierkens $^{1}$, J.J. Boelens ${ }^{2}$, N. van Til ${ }^{1}$

${ }^{1}$ UMC Utrecht, LTI-Applied, Utrecht, The Netherlands

${ }^{2}$ MSK-Cancer Center, Pediatrics, New York, USA

Hematopoietic stem cell transplantation (HSCT) can potentially cure pediatric patients with acute myeloid leukemia (AML). However, a large proportion of transplanted patients eventually die due to relapse. To improve overall survival, we propose a combined strategy based on the use of cord blood (CB) derived cells for cellular therapy and HSCT. We generated CB-CD8+ $\mathrm{T}$ cells expressing a recombinant $\mathrm{T}$ cell receptor (TCR) against Wilms tumor 1 (WT1) lacking endogenous TCR expression in order to avoid mispairing and competition. These cells were created through a highly efficient CRISPR-Cas9 multiplexing approach (up to $95 \%$ gene-editing efficiency) targeting the endogenous TCR alpha or beta chain. Next to this strategy, high transduction efficiencies (more than 80\%) and expression levels were obtained with lentiviral constructs containing viral and housekeeping gene promoters driving the expression of $\mathrm{WT} 1_{126}$-TCR. By combining these approaches, we developed a tumor antigen-specific $\mathrm{T}$ cell product with robust expression of a WT1 $1_{126}$-specific TCR and very efficient elimination of endogenous TCR expression, to kill WT-1 expressing tumor cells. Gene-edited $\mathrm{T}$ cells show enhanced proliferation rate and cytokine production upon stimulation and express low levels of exhaustion markers (such as PD1, LAG3 and TIM3). Further product development could provide a potent immune cell therapy approach to be used after HSCT to eliminate residual AML blasts and increase overall survival chances in pediatric relapse patients.

\section{ICLEa19-0030}

Hematological Malignancies

\section{REPROGRAMMED CAR T CELLS MAINTAIN METABOLIC POTENTIAL DURING RAPID EXPANSION AND SHOW INCREASED ANTI-TUMOR REACTIVITY}

\author{
K. Mølgaard ${ }^{1}$, Ö. Met $^{1}$, I.M. Svane ${ }^{1}$ \\ ${ }^{1}$ Center for Cancer Immune Therapy, Department \\ of Hematology, Herlev Hospital, Herlev, Denmark
}

Introduction: Chimeric antigen receptor (CAR) T cell therapy has proven to be highly successful in hematological cancers and are now being tested in various solid tumors. Recent data support a less differentiated cytotoxic $\mathrm{T}$ cell subpopulation to be critical for long-term persistence and survival. Standard, rapid expansion using high dose IL- 2 and $\mathrm{CD} 3 \times \mathrm{CD} 28$ stimulation causes later differentiation and short-lived $\mathrm{T}$ cells1. Consequently, changing expansion conditions increases the anti-tumor activity and the frequency of long-lived memory $\mathrm{T}$ cells. Considering this, we have screened alternative options for cytokine supplementation in rapid expansion of CAR T cells and evaluated the differentiation pattern based on surface markers and metabolic potential along with anti-tumor reactivity to improve CAR T cell products. Results and conclusion: Standard rapid expansion using IL-2, results in a 2000-fold expansion of cells within a few weeks. By changing cytokine supplement to IL-21 CAR T cells are detained in a less differentiated state, but at the cost of fold-expansion. Rapid expansion using IL-7 or IL-15 produce a less exhausted cell product while still maintaining fold-expansions similar to conventionally expanded cells. IL-7 expanded CART T cells produce higher amount of anti-tumor related cytokines compared to IL- 2 and IL-15 expanded cells in ICS. Furthermore, metabolic analysis of IL-21 expanded CARs seem to have low metabolism and high spare capacity consistent with a naïve-like phenotype where IL-2 expanded CARs have high metabolic demands and low spare capacity. Interestingly, IL-7 expanded CARs have metabolic capacities comparable to IL-21 expanded cells, but still rapidly expand in culture to high cell numbers. Changing the cytokine stimulation in rapid expansion has a positive impact on the differentiation and the metabolic potential for CAR T cells while still maintaining high fold-expansions. These metabolically reprogrammed CAR $\mathrm{T}$ cells show improved anti-tumor reactivity and could potentially outperform conventual CART cells in vivo.1Klebanoff, C.A. et al. (2004)

\section{ICLEa19-0035 \\ Hematological Malignancies}

\section{HEMATOPOIETIC MINOR HISTOCOMPATIBILITY ANTIGEN-SPECIFIC CD8+ T CELLS FOR ANTI- RELAPSE THERAPY AFTER ALLOGENEIC HEMATOPOIETIC STEM CELL TRANSPLANTATION}

\author{
A. Pilunov ${ }^{1}$, D. Romaniuk ${ }^{1}$, S. Sheetikov ${ }^{1}$, \\ A. Khmelevskaya ${ }^{1}$, A. Shmelev ${ }^{1}$, N. Bykova ${ }^{1}$, \\ A. Shomuradova ${ }^{1}$, D. Kiryukhin ${ }^{1}$, D. Dianov ${ }^{1}$, G. Efimov ${ }^{1}$ \\ ${ }^{I}$ National Research Center for Hematology, Laboratory \\ of Transplantation Immunology, Moscow, Russia
}

Relapse of acute leukemia occurs in one-third of the patients after fully HLA-matched allogeneic stem cell transplantations (allo-HSCT) causing high mortality. There is evidence that graft 
versus leukemia reaction is promoted by the immune response to minor histocompatibility antigens (MiHAs) - endogenous polymorphic peptides, presented by MHC. MiHAs ACC-1Y and HA-1 are presented by the frequent HLA alleles and encoded by the genes predominantly expressed in the hematopoietic tissue, thus they represent the clinically relevant targets in postHSCT relapse.

Naïve CD8+ T cells from MiHA-negative donors were expanded in vitro using autologous dendritic cells, pulsed with MiHA peptides. Antigen-specific cells were sorted and their T-cell receptor (TCR) alpha and beta chains were sequenced. We have selected one ACC-1Y-specific and five HA-1-specific TCRs for further analysis.

Lentiviral transduction of CD8+ lymphocytes with ACC-1Yspecific TCR confirmed its specificity to the target antigen.

We have designed a novel lentiviral backbone for rapid TCR cloning and further functional screening of a panel of HA-1specific alpha and beta chains, based on the Golden Gate Assembly. This backbone allows switching between selective markers, here we used the truncated form of CD34.

To prevent off-target effects caused by mispairing of endogenous and introduced TCR chains in modified cells, endogenous TCR knockout strategy was designed by transfection of CD8+ cells with gRNA/Cas9 ribonucleoprotein complexes prior to transduction. At the same time, transgenic alpha and beta-chain constant domains were modified to be resistant to Cas9 cleavage and extra cysteines were introduced for TCR stability. HLAA*02:01 lymphoblastoid B-cell lines presenting HA-1 will be used to confirm the specificity and test the functional avidity of the transduced cells by IFN- $\gamma$ secretion assay.

Adoptive transfer of MiHA-specific genetically modified $\mathrm{T}$ cells from HLA-matched and MiHA-mismatched donor may be used to treat leukemia relapse after allo-HSCT. The work was supported by the Russian Science Foundation grant 17-15-01512.

\section{ICLEa19-0041}

\section{New Horizons in Lympocyte Engineering}

\section{IN VIVO THYMOCYTE ENGINEERING}

N. Gritsenko $^{1}$, M. Fried ${ }^{1}$, C. Pundak-Mintz ${ }^{1}$, Y. Zilberstein ${ }^{2}$, I. Dotan $^{1}$, A. Zoaby ${ }^{1}$, A. Globerson Levin ${ }^{3}$, A. Barzel ${ }^{1}$

${ }^{1}$ Tel Aviv University, Biochemistry and Molecular Biology, Tel aviv, Israel ${ }^{2}$ Tel Aviv University, Sackler Cellular and Molecular Imaging Center, Tel aviv, Israel ${ }^{3}$ Tel Aviv Sourasky Medical Center TASMC, Immunology research center, Tel aviv, Israel

Chimeric antigen receptor (CAR) T cells have shown promising results in patients with B-cell malignancies and other types of cancer. However, present procedures for the treatment are cumbersome and time consuming, which can be detrimental to the patient. Here, we propose a new method of in vivo $\mathrm{T}$ cell engineering that may circumvent these challenges and achieve the desired effects of treatment directly inside the body, by an injection of a CAR-coding vector. Delivery of the therapeutic gene is provided by recombinant Adeno Associated Virus (rAAV) that is used in FDA approved therapeutics and allows lymphocyte engineering. Additionally, for stable therapeutic gene expression, we propose a novel immunotherapy approach directing immune genes into the VDJ locus of developing T cells using the natural VDJ recombination process. Our preliminary results demonstrate the feasibility of using rAAV or the jetPEI reagent to deliver DNA into the T cells in-vivo, by intra-thymic injections. Moreover, upon intra-thymic AAV transduction, we show integration of the transgene into the VDJ locus, by the naturally expressed RAG complex within highly potent developing $\mathrm{T}$ cells.

\section{ICLEa19-0048}

New Horizons in Lympocyte Engineering

\section{ADVANCING CAR T CELL THERAPY UTILIZING IN SITU TRANSDUCTION}

\author{
G. Horn $^{1}$, A. Najjar ${ }^{2}$, A. Globerson Levin ${ }^{1}$, T. Waks ${ }^{1}$, Z. Eshhar ${ }^{1}$ \\ ${ }^{1}$ The Tel Aviv Sourasky Medical Center, Immunology lab, Tel \\ Aviv, Israel ${ }^{2}$ The University of Texas M.D. Anderson Cancer \\ Center, Department of Pediatrics, Houston- Texas, USA
}

Introduction: Chimeric antigen receptor (CAR) cancer therapy uses $\mathrm{T}$ cells reprogrammed with antitumor specificity. However, the complexity and high cost associated with individualized production of CAR $\mathrm{T}$ cells are factors that limit extending this therapeutic approach to a greater number of patients. Our goal is to develop lentiviral vectors that specifically target endogenous $\mathrm{T}$ cells to redirect their specificity toward tumor antigens.

Methods: As a first step, we have constructed several lentiviral vectors prototypes that can specifically target $\mathrm{T}$ cells to deliver and express erbB-2-specific CAR. The constructs consisted of $\mathrm{scFv}$ derived from different Her2-specific antibody recognition domain fused to CD28 and FcRgamma or 4-1BB. Pseudotype receptors were designed by fusing $\mathrm{scFv}$ regions specific for mouse (2C11) and human (OKT3) CD3 with fulllength vesicular stomatitis virus glycoprotein (VSVG) or the transmembrane domain of VSVG (VTMD). Lentiviral vectors expressing these surface pseudotypes and encoding a firefly luciferase (ffLuc) reporter gene were tested in vitro. In situ transduction efficiencies were assessed in two different mouse models. The human CD3-targeting pseudotyped (OKT3-VTMD) lentivirus encoding ffLuc was tested in humanized NSG mice. The transduction efficiency of the mouse CD3-specific pseudotypes were assessed in immunocompetent BALB/c mice. Both mouse models have shown in situ transduction in a period of up to 7 days post injection and in regions consistent with $\mathrm{T}$ cell localization such as bone marrow and spleen. One week following intravenous infusion of lentiviral particles, GFP expression was detected in the $5-30 \%$ of CD3+ cells

Conclusion: We have demonstrated the feasibility of transducing endogenous $\mathrm{T}$ cells in situ in humanized and in immunocompetent mouse models. We believe that in situ transduction will have a significant impact in advancing the field of CAR T cell therapy by addressing the current limitations associated with distribution, accessibility, and cost of adoptive cell therapy.

\section{ICLEa19-0039}

New Horizons in Lympocyte Engineering

\section{ENGINEERING B CELLS FOR A REGULATED, IMMUNODOMINANT AND EVOLVING TREATMENT FOR HIV INFECTIONS}

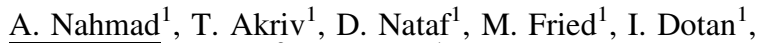 \\ Y. Wine ${ }^{2}$, I. Benhar ${ }^{2}$, A. Barzel ${ }^{1}$ \\ ${ }^{1}$ Tel Aviv University, Biochemistry and Molecular Biology, \\ Tel Aviv, Israel ${ }^{2}$ Tel Aviv University, Department Molecular \\ Microbiology and Biotechnology, Tel Aviv, Israel
}


Broadly Neutralizing antibodies (bNAbs) are an effective class of antibodies against HIV and their administration to infected individuals is associated with good clinical outcomes. However, high cost of treatment and repeated injections are predictable. Moreover, viral escape limits the therapeutic effect of injected bNAbs.

Using CRISPR/Cas9 and Adeno Associated Vectors (AAV), we introduce the anti-HIV broadly neutralizing antibody 3BNC117 at the immunoglobulin heavy chain (IgH) locus of B cells. The light chain is encoded in full followed by the variable segment of the heavy chain, separated by a 2 A peptide. Downstream of the variable heavy chain, we inserted a splice donor, which allows splicing with the endogenous constant segment.

Adoptive transfer of the engineered cells into syngeneic mice allowed antigen-induced activation upon immunization. Donor specific cells were tracked with the CD45.1 cell surface marker in CD45.2 recipient mice. In germinal centers (GC) following immunization, all donor specific cells were binding to the HIV antigen gp120, demonstrating the strong selection for the specific humoral response. Strikingly, most of the gp120 binding cells in GCs were donor derived, demonstrating immunodominance of the engineered cells over the endogenous natural response. Sequencing of the transgene during GC response showed patterns of SHM, implying affinity maturation. We followed the serological response using an anti-idiotypic antibody to quantify antibody titers. Interestingly, some of the total bNAb response would underdo CSR in-vivo, as demonstrated by $\operatorname{IgA}$ isotype in serum and in GCs. The total antibody response rose much further following boost immunization implying immunological memory and affinity maturation.

Uniquely, our method enables antigen-induced bNAb secretion that may be further enhanced by affinity maturation, class switch recombination, and the retention of immunological memory. B cells could thus be engineered as a living and evolving drug to counteract pathogen escape.

\section{ICLEa19-0015}

New Horizons in Lympocyte Engineering

\section{TOWARDS SAFE AND EFFICIENT DELIVERY OF MRNA AND CRISPR-CAS9 COMPLEXES IN T CELLS BY VAPOR NANOBUBBLE PHOTOPORATION}

L. Raes $^{1}$, S. Stremersch ${ }^{1}$, J. Fraire ${ }^{1}$, T. Brans ${ }^{1}$, G. Goetgeluk ${ }^{2}$, J. Van Hoeck ${ }^{1}$, R. Xiong ${ }^{1}$, B. Vandekerckhove ${ }^{2}$, S. De Smedt C. ${ }^{1}$, K. Raemdonck ${ }^{1}$, K. Braeckmans ${ }^{1}$

${ }^{1}$ Ghent University, Department of Pharmaceutics, Ghent, Belgium ${ }^{2}$ Ghent University, Department of Clinical ChemistryMicrobiology and Immunology, Ghent, Belgium

Adoptive $\mathrm{T}$ cell therapy is a rapidly emerging approach in cancer immunotherapy, harnessing the patients' own immune cells to fight cancer. Modification of $\mathrm{T}$ cells is most often performed by viral transduction or electroporation, but their use is typically restricted by cytotoxicity and safety issues. Vapor NanoBubble (VNB) photoporation has recently gained interest as a widely applicable technique for transfection of macromolecules and was already shown successful for siRNA delivery to murine T cells [1]. In this study, we evaluate whether VNB photoporation could serve as a safe technology for mRNA delivery.
For VNB photoporation, gold nanoparticles (AuNPs) are attached to the cell membrane and subsequently illuminated with a pulsed laser. VNBs arising from the AuNPs lead to the formation of transient pores and allow direct delivery of mRNA. We carefully optimized a multitude of parameters (e.g. AuNP concentration) related to the VNB-photoporation procedure for mRNA transfection of both HeLa and Jurkat T cells. In addition, we compared the VNB-photoporation technology with Nucleofection ${ }^{\mathrm{TM}}$, i.e. the current standard technique for $\mathrm{T}$ cell transfection.

For HeLa cells, we reached up to $40 \%$ transfected cells while maintaining high cell viability, using DPBS+ ${ }^{\circledR}$ as transfection buffer and $1.5 \mu \mathrm{M}$ mRNA. Most interestingly, we observed a threefold increase in positive living cell yield for Jurkat $\mathrm{T}$ cells by VNB photoporation, compared to Nucleofection ${ }^{\mathrm{TM}}$. In conclusion, VNBphotoporation represents a promising, safe and efficient technology for mRNA transfection of Jurkat $\mathrm{T}$ cells. Current efforts aim to investigate its applicability for delivery of mRNA and CRISPRCas9 complexes to primary human $\mathrm{T}$ cells. If successful, the technology holds great promise for generation of allogeneic universal CAR-T cells.

\section{ICLEa19-0023 Other \\ SYNTHETIC SURFACES FOR ENHANCEMENT OF T-CELL THERAPIES}

\author{
S. Adutler-Lieber ${ }^{1}$, N. Friedman ${ }^{1}$, B. Geiger ${ }^{2}$ \\ ${ }^{1}$ Weizmann Institute of Science, Immunology, Rehovot, Israel \\ ${ }^{2}$ Weizmann Institute of Science, Molecular Cell Biology, \\ Rehovot, Israel
}

Adoptive immunotherapy is based on ex vivo expansion and stimulation of T-cells, followed by their transfer into patients. The need for the ex vivo culturing step provides opportunities for modulating the properties of transferred T-cells, enhancing their antitumor abilities, and increasing their expansion.

We have developed synthetic surfaces that increase the proliferation and antitumor activity of T-cells. We first evaluated the effect of various compositions that mimic the physiological microenvironment encountered by T-cells during their activation and expansion in the lymph node.

We found that substrates coated with the chemokine CCL21 together with the adhesion molecule intercellular adhesion molecule 1 (ICAM1) significantly increase the proliferation of $\mathrm{CD}^{+} / \mathrm{CD}^{+}$mouse and human T-cells, activated by either antigen-loaded dendritic cells or activation microbeads. Notably, antigen-specific $\mathrm{CD}^{+} \mathrm{T}$-cells, cultured on these substrates, also displayed augmented cytotoxic activity toward antigen-matching tumor cells, both in-vitro and in-vivo. This increase in specific cytotoxic activity was associated with a major increase in the cellular levels of the killing-mediator granzyme B.

While the addition of soluble IL-6 to T-cells cultures on CCL21+ICAM1 coated surfaces significantly increased T-cell viability and expansion, it in fact impaired T-cells functionality and cytotoxicity, demonstrating possible independent effect on expansion and function.

Our results suggest that these surfaces may be used for shortening expansion time and generation of T-cells with augmented cytotoxic function, for use in cancer immunotherapy. 


\section{ICLEi19-0008}

Other

\section{RECEPTORS PROVIDING TUMOUR ANTIGEN RESTRICTED COSTIMULATION ENHANCE T-CELL ACTIVITY FOR ADOPTIVE T-CELL THERAPY}

\author{
M. Sykorova ${ }^{1}$, M. Kalaitsidou ${ }^{1}$, M. Le Brocq ${ }^{1}$, A. Patel ${ }^{1}$, \\ T. Katopodi ${ }^{1}$, R. Hawkins ${ }^{1}$, G. Kueberuwa ${ }^{1}$, J. Bridgeman ${ }^{1}$ \\ ${ }^{1}$ Immetacyte Ltd., Research \& Development, Manchester, United \\ Kingdom
}

Tumour infiltrating lymphocyte (TIL) therapy involves the ex vivo culture and expansion of tumour derived lymphocytes, in particular T-cells, before readministration to the same patient. A number of centres around the world have shown remarkable success using TIL therapy for treatment of metastatic melanoma; furthermore, trials in other cancer indications have shown encouraging results.

T-cell activation requires a 'signal 1' through the engagement of TCR with pMHC and a second costimulatory pathway termed 'signal 2' which enhances T-cell proliferation and survival; however, tumours rarely express the ligands which provide the 'signal 2 ', potentially leading to an ineffective anti-tumour response. We have therefore investigated whether recombinant fusion receptors consisting of a costimulatory domain and a tumour antigen recognition domain, so called costimulatory antigen receptors (CoStAR), can artificially costimulate T-cells upon TCR engagement.

We have generated a prototype receptor to demonstrate this effect and undertaken extensive engineering strategies to identify an optimum signalling domain. Next, we turned our attention to the antigen binding domain, developing recognition domains which specifically permit targeting of defined tumour types. To this end we have optimised CoStARs targeting colorectal, ovarian and renal cancer.

These CoStARs not only enhance cytokine production by engineered T-cells, but also enhance survival and proliferation, thus providing a rationale to engineer TIL in an effort to enhance treatment efficacy.

\section{ICLEi19-0010 \\ Other}

\section{NANOSTRAW-MEDIATED DELIVERY ENABLES FAST AND EFFICIENT DELIVERY OF MOLECULAR CARGO TO HARD-TO-TRANSFECT MAMMALIAN CELLS IN A NON-PERTURBATIVE FASHION WHILE MAINTAINING HIGH VIABILITY}

S. Johnson $^{1}$, S. Leal-Ortiz ${ }^{1}$, M. Hjort ${ }^{1}$, H. Vossfeldt ${ }^{1}$, $\overline{\text { R. Swoboda }}^{1}$, S. Boyatzis ${ }^{1}$

${ }^{1}$ Navan Inc, Navan Inc, South San Francisco, USA

The redirection of antigen specificity of T-cells via the transfer of desired antigen receptor genes and the adoptive transfer of these genetically designed T-cells into patients holds excellent promise for individually tailored treatment of cancerous diseases. Similarly, the development of new technologies like CRISPR/Cas9, other editing nucleases and next-generation transposons present exciting options to treat myriad diseases. In each of these novel therapeutic approaches, it is vital for cells to be altered by the delivery of a cargo to a target cell, which is often problematic if the cargo is large or complex, or the cell is sensitive, like immune cells are. A powerful intracellular delivery method should facilitate an effective and convenient means of cell transfection while also being cost-effective, easily implementable and scalable for clinical applications. In addition to these features, the ideal approach would leave the transfected cells happy and healthy (preventing any perturbation of the cells) and maximize the chance of survival throughout the end-to-end process.

The NanoStraw technology achieves rapid delivery to enable high throughput methods and is capable of delivering diverse cargoes such as DNA, RNA and protein, directly to the cells without causing high cell death (per traditional electroporation methods), or immunological changes observed with lentiviral transduction. Herein, we show that primary $\mathrm{T}$ cells have a viability of $98 \%$ forty-eight hours post-delivery, when using industry standard luciferase reporter assays to accurately measure cell death. Furthermore, we demonstrate high efficiency in multiple types of hard-to-transfect cells including Jurkat cells, primary lymphocytes and CD34+ stem cells, delivering various cargoes while maintaining cellular integrity and viability. NanoStraws present a robust method for intracellular delivery of virtually any cargo into hard-to-transfect cells and consequently, can fulfill the need for a gentle, capable, scalable, universal, dosage-controlled, and cost-effective platform in the development of next-generation cell and gene therapies.

\section{ICLEa19-0051 \\ Other}

\section{THE ROLE OF THE EXTRACELLULAR CD28 DOMAIN IN CAR T CELLS: MONOMERISATION AND TRUNCATION IMPACT THE ACTIVATION OF CD28 CAR T CELLS}

A. Kilgallon $^{1}$, R. Hawkins ${ }^{1}$, E. Cheadle ${ }^{1}$, J. Bridgeman ${ }^{1}$, A. Armstrong ${ }^{1}$, D. Gilham ${ }^{1}$, A. Schutt ${ }^{1}$, M. Kalaitsidou ${ }^{1}$, G. Kueberuwa ${ }^{1}$

${ }^{1}$ University of Manchester, Division of Cancer Sciences, Manchester, United Kingdom

Within the CAR T cell field, much effort is placed upon discovery of the optimal co-stimulatory domain, yet few studies focus on specific domains within co-stimulatory receptors.

The current study aimed to elucidate specific aspects of the extracellular domain of CD28 in a second generation CEAspecific CAR. CD28 has a co-stimulatory function, activated by binding of the $\mathrm{B} 7$ molecule ligands through a conserved motif (MYPPPY). Endogenous CD28 is also a homodimer. We therefore investigated the role of CD28 CAR dimerisation and CD80/86 interactions upon CAR T cell activation.

To assess the role of co-stimulatory binding, the conserved B7 binding motif was fundamental; this motif was mutated to generate a new CAR (mut.CD28). Two further CARs were generated by truncation of the extracellular domain of the CD28 molecule, either upstream (trU.CD28) or downstream (trD.CD28) of the binding motif. All three of these CARs displayed loss of binding to B7-1 and B7-2. It was observed that CAR truncation negatively impacted CAR expression; whereas expression of the mutCD28 CAR was comparable to the parental CD28 CAR.

To assess the role of dimerisation within CD28 CARs, a monomeric version of the mutCD28 receptor was generated (monoCD28) these failed to dimerise and existed as monomers on the $\mathrm{T}$ cell surface. The expression of this CAR compared to the mutCD28 was dramatically reduced, implicating dimerisation in surface expression levels.

Functional testing of these CAR $\mathrm{T}$ cells, demonstrated a positive correlation between CAR surface expression and $\mathrm{T}$ cell activity. Parameters measured include production of lymphoproliferative and cytotoxic cytokines and $\mathrm{T}$ cell degranulation 
upon stimulation through the CAR. Further investigations will include measuring phosphorylation of downstream signalling molecules upon CAR stimulation. The correlation between T cell activity and CAR expression is currently under investigation.

\section{ICLEa19-0009 \\ Other}

\section{MELANOMA-INFILTRATING DENDRITIC CELLS ARE REQUIRED FOR LICENSING THE CYTOTOXIC ACTIVITY OF CD8+ T CELLS IN SITU}

N. Santana-Magal $^{1}$, L. Farhat-Younis ${ }^{1}$, D. Rasoulouniriana ${ }^{1}$, A. Gutwillig ${ }^{1}$, L. Tal $^{1}$, P. Rider ${ }^{1}$, Y. Carmi ${ }^{1}$

${ }^{1}$ Tel Aviv University, Department of Pathology, Sackler School of Medicine, Tel Aviv, Israel

The recent success of checkpoint blockade therapies has established immunotherapy as one of the most promising treatments for melanoma. Nonetheless, a complete curative response following immunotherapy is observed only in a fraction of the patients. To understand the factors that limit the efficacy of immunotherapies, we established mouse models which cease to respond to immunotherapies once tumors exceed a certain stage. We compared the changes within immune cell populations in the tumor, draining lymph node (DLN) and blood, in responders and non-responding melanoma tumor-bearing mice. Analysis of their immune system revealed that the numbers of tumor-infiltrating DC (TIDC) drastically decrease with time. We further found that in contrast to the current paradigm, once melanoma is established, TIDC do not migrate into sentinel lymph nodes. Instead, these DC undergo local cell death due to excessive phagocytosis of melanosome. Importantly, we found that TIDC are required to license the cytotoxic activity of tumor CD8+ T cells, and in their absence, T cells will not lyse melanoma cells. Overall, this research provides a new insight into the roles of TIDC in tumor, and the means to rescue them from melanosomes-induced apoptosis. Thereby increasing the efficacy of checkpoint blockades, adoptive $\mathrm{T}$ cell transfer, as well as other $\mathrm{T}$ cell-based immunotherapies.

\section{ICLEa19-0037}

\section{TCR Targeting Solid Tumors}

IDENTIFICATION OF A T CELL RECEPTOR SPECIFIC FOR GLYPICAN 3 FOR ADOPTIVE IMMUNOTHERAPY

$\underline{\text { S. Hervas-Stubbs }}{ }^{1}$, J. Gonzalez-Vaz ${ }^{1}$, U. Mancheno ${ }^{1}$, E. Elizalde $^{1}$, I. Tamayo ${ }^{1}$, P. Sarobe ${ }^{1}$

${ }^{1}$ Center for Applied Medical Research CIMA- University of Navarra, Immunology and Immunotherapy, Pamplona, Spain

Immunotherapy with $\mathrm{T}$ lymphocytes genetically modified to express tumor-specific T-cell receptors (TCRs) may be an effective treatment for cancer patients non-responder to conventional therapies and immunecheckpoint blockades. An interesting target for TCR therapy is Glypican 3 (GPC3). GPC3 is expressed by several types of tumors, including hepatocellular carcinomas (HCC), ovarian cancer, malignant melanoma, and testicular yolk sac tumor, but not in healthy tissues. T cells specific for
GPC3 have been detected in HCC patients. Central tolerance and/or exhaustion of $\mathrm{T}$ cells in cancer patients could be an obstacle to find high-affinity anti-GPC3 TCRs in humans. In order to identify TCRs of optimal affinity, in this study we have used the murine HHD model expressing the HLA-A2 molecule. Using single-cell NGS, we have identified HLA-A2-restricted GPC3-specific TCRs from HHD mice immunized with human GPC3. Functionality and specificity of the isolated TCRs toward HLA-A2+GPC3+ tumor cell lines will be subsequently investigated in vitro and in vivo by transduction of human $\mathrm{T}$ cells. The GPC3-specific TCR genes identified in this study may have a great potential to engineer patient's autologous $\mathrm{T}$ cells for the treatment of various neoplasms.

Funding sources: ISCIII/FEDER, EU (PI18 / 00556)

ICLEa19-0042

TCR Targeting Solid Tumors

\section{A NOVEL TCR CLONING SYSTEM OF PEPTIDE- SPECIFIC T CELLS USING IMMUNOSPOT ARRAY ASSAY ON A CHIP (T-ISAAC) TECHNOLOGY}

E. Kobayashi ${ }^{1}$, T. Ozawa ${ }^{1}$, H. Hamana ${ }^{1}$, K. Shitaoka ${ }^{1}$, A. Muraguchi $^{1}$, H. Kishi ${ }^{1}$

${ }^{1}$ University of Toyama, Department of Immunology, Toyama-shi, Japan

The T cell receptor (TCR) gene therapy is expected as a next generation therapy for tumor as well as infectious diseases. Thus, an effective screening system for analyzing both Ag-specific TCR alpha/beta pairs from single T cells is requisite.

Recently we developed a microwell array chip technology that enabled us direct identification of antigen-specific antibodysecreting cells and efficient and rapid production of human monoclonal antibody (immunospot array assay on a chip, ISAAC). Using the microarray chip, we have recently found that TCRs and peptide/MHC on the cell surface of a single CD8+T cell can interact (as a manner of cis-interaction) and induce cytokine secretion. In this study, we applied the ISAAC technology to detect antigen-specific T-cells and clone their TCR cDNAs (T-ISAAC).

To detect antigen-induced cytokine-secretion on the chip, we prepared CD8+T cells from HLA-A24(+) healthy donors, arrayed them on an anti-IFN- $\gamma$ antibody-coated chip, and stimulated them with HLA-A*2402 Epstein-Barr virus EBNA3A peptide with anti-CD28 antibody. After 6 hours, we detected antigen-specific $\mathrm{CD} 8+\mathrm{T}$ cells that secreted IFN- $\gamma$ using fluorescence-labeled anti-IFN- $\gamma$ antibody. We then retrieved the IFN- $\gamma$-secreting cells from the chip and amplified cDNA of TCR alpha and beta chain using single-cell RT-PCR. Thereafter, we transfected both the alpha- and beta-chain expression vectors in a TCR-T cell line. Flow cytometric analysis revealed that the cloned TCRs bound to EBNA3A-peptide/MHC tetramer. All process was performed within 10 days. These results show that T-ISSAC technology could detect antigen-specific $\mathrm{T}$ cells and yield antigen-specific TCRs efficiently. This novel method enables us detection of antigen-specific $\mathrm{T}$ cells and rapid cloning of their TCRs, providing a powerful tool for screening the TCRs for TCR gene therapy.

This research was supported by AMED under Grant Number JP17cm0106417h0002. 


\section{ICLE 2019-Poster Presentations}

\section{ICLEa19-0013}

CARs Targeting Solid Tumors

\section{A NOVEL UNIVERSAL CAR T CELLS IMMUNOTHERAPY FOR THE TREATMENT OF SOLID TUMORS}

L. Farhat-Younis $^{1}$, D. Rasoulouniriana ${ }^{1}$, N. Santana-Magal ${ }^{1}$,

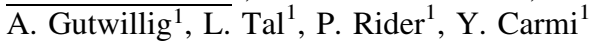

${ }^{1}$ Sackler School of Medicine-Tel Aviv University, Pathology, Tel Aviv, Israel

Recently, remarkable clinical responses were observed in patients treated with engineered $\mathrm{T}$ cells, establishing immunotherapy as one of the most promising clinical approaches for cancer. Nonetheless, for unknown reasons, therapies using engineered $\mathrm{T}$ cells are currently effective only with hematological malignancies, and treated patient often suffer a relapse. Furthermore, the lack of identified tumor-specific antigens limits the clinical use of this therapy and often results in on-target, off-site toxicity. Therefore, an urgent need remains to develop treatments capable of eradicating solid tumors, which feature a higher safety profile and do not depend exclusively on the host $\mathrm{T}$-cell repertoire.

In an unpublished study, we have recently discovered a novel subset of $\mathrm{CD}^{+} \mathrm{T}$ cells that directly kill tumor cells coated with antibodies. These cells express the high affinity Fcg receptor (FcgRI), which enables them to bind coated tumor cells and secrete lytic granules resulting in remarkable tumor lysis. Recognition of the tumor also involves engagement of their $\mathrm{T}$ cell receptor (TCR), thus providing another lair of specificity. Importantly, we were able to recapitulate the cytotoxic capacities of that population in conventional $\mathrm{CD}^{+} \mathrm{T}$ cells by co-infecting them with FcgRI and the FcR common gamma chain. Indeed, these FcgRI engineered T cells exerted remarkable killing capabilities of solid tumors, upon incubation with antibodies. Unlike chimeric-antigen receptor $\mathrm{T}$ (CAR-T) cells, which require chaining the targeting receptor with each tumor, this technology can be applied to treat a wide range of cancers by using clinically-approved antibodies, or antibodies with a high safety profile that have failed in phase III clinical trials.

\section{ICLEa19-0036}

CARs Targeting Solid Tumors

\section{REDIRECTION OF UNICAR T CELL AGAINST EGFR+ TUMOR CELLS BY USING DIFFERENT $\alpha$ EGFR TARGETING MODULE FORMATS}

\author{
J. Jureczek $^{1,2}$, A. Feldmann ${ }^{3}$, S. Albert ${ }^{3}$, R. Bergmann ${ }^{3}$, \\ N. Berndt ${ }^{1}, 3$, C. Arndt ${ }^{3}$, S. Koristka ${ }^{3}$, M. Bachmann ${ }^{1,2,3,4}$ \\ ${ }^{1}$ German Cancer Consortium DKTK- partner site Dresden, \\ German Cancer Research Center DKFZ- Heidelberg- Germany, \\ Dresden, Germany ${ }^{2}$ University Cancer Center UCC, Tumor \\ Immunology- University Hospital Carl Gustav Carus- TU \\ Dresden, Dresden, Germany ${ }^{3}$ Institute of Radiopharmaceutical \\ Cancer Research, Helmholtz-Zentrum Dresden-Rossendorf \\ HZDR, Dresden, Germany ${ }^{4}$ National Center for Tumor Diseases \\ NCT-partner site Dresden, University Hospital Carl Gustav \\ Carus- TU Dresden, Dresden, Germany
}

Since epithelial growth factor receptor (EGFR) mutations or overexpression is linked with variety of malignancies, including lung, breast, stomach, colorectal, head and neck carcinomas as well as glioblastomas, it is an attractive target for tailored treatment. As chimeric antigen receptor (CAR) engineered $\mathrm{T}$ cells highly effectively eliminate hematological malignancies already in the clinics, one idea is to redirect CAR T cells also against EGFR expressing solid cancers. However, CAR T cell therapy can lead to severe, even life-threatening side effects with high risk of on-target/off-tumor activity. To overcome these challenges our UniCAR technology might be an appropriate answer combining high anti-tumor effectiveness, tumor specificity, flexibility, and safety control mechanisms. In contrast to conventional CARs, UniCAR T cells are per se inert because UniCARs are directed against a small peptide epitope, which is not present on living cells. The redirection of UniCAR T cells toward target cells occurs only in the presence of a tumor specific targeting molecule (TM) - bifunctional moieties carrying the specificity for a certain tumor antigen and contain the UniCAR peptide epitope recognized by UniCARs. TMs can be made of different molecules showing various structures. Here we are presenting the comparison in functionality, in vitro and in vivo, of two TM formats: nanobody based $\alpha \mathrm{EGFR} T \mathrm{TM}$ derived from the camelid $\alpha$ EGFR antibody $7 \mathrm{C} 12$ and scFv derived TM from the clinically used chimeric monoclonal antibody Cetuximab (IMC C-225). In principle, we observed that both TM formats are able to redirect UniCAR $\mathrm{T}$ cells to eliminate EGFRexpressing tumor cells in an antigen-specific and TM-dependent manner. Most interestingly, the tumor killing efficiency of the $\alpha$ EGFR scFv TM was significantly superior in comparison to the nanobody based TM, what might decide whether UniCAR T cells attack target cells showing different EGFR density level.

\section{ICLEa19-0024}

CARs Targeting Solid Tumors

\section{A THERANOSTIC DRUG FOR PROSTATE CANCER CAR T CELL IMMUNOTHERAPY AND PET IMAGING}

C. $\operatorname{Arndt}^{1}, \underline{\text { S. Koristka }}{ }^{1}$, N. Berndt ${ }^{1}$, R. Bergmann ${ }^{1}$, L. Loureiro $^{1}$, J. Kotzerke ${ }^{2}$, M. Bachmann ${ }^{1}$, A. Feldmann ${ }^{1}$

${ }^{1}$ Helmholtz-Zentrum Dresden-Rossendorf HZDR, Institute

of Radiopharmaceutical Cancer Research, Dresden, Germany

${ }^{2}$ University Hospital 'Carl Gustav Carus' TU Dresden,

Department of Nuclear Medicine, Dresden, Germany

Although chimeric antigen receptor (CAR) $\mathrm{T}$ cells have demonstrated outstanding therapeutic efficacy, current design of clinically approved CAR constructs renders therapies monospecific, inflexible and imperiled to severe side effects. We therefore refined the CAR approach and established a switchable platform technology termed UniCAR. Instead of directly interacting with tumor cells, UniCAR-engrafted T cells recognize the short peptide epitope E5B9. Their on/off switch is mediated by E5B9-comprising targeting modules (TMs), facilitating indirect cross-linkage to antigen-expressing cancer cells.

As all TMs described to date contain an antibody-derived binding moiety, we aimed to develop a ligand-based low-molecular 
weight agent that enables not only UniCAR T cell retargeting, but also monitoring of the therapeutic response by non-invasive PET imaging.

For proof of concept of this novel theranostic approach, we successfully converted the clinically approved radiotracer PSMA-11 into a TM by fusion with E5B9 without affecting its diagnostic properties in mice and humans. Moreover, the chemically synthesized, ligand-based compound efficiently mediated PSMA-expressing tumor cell lysis by UniCAR T cells both in vitro and in vivo.

Taken together, the PSMA-ligand TM represents a novel theranostic agent that is an attractive candidate for immunotherapy of prostate cancer as well as for initial diagnosis and follow-up treatment.

\section{ICLEa19-0012}

\section{CARs Targeting Solid Tumors}

\section{A NOVEL SUBSET OF CD4+ T CELL EXPRESSING THE HIGH AFFINITY Fc $\gamma$ RECEPTOR LINKS ANTIBODY AND T CELL IMMUNITY}

D. Rasoulouniriana ${ }^{1}$, N. Santana-Magal ${ }^{1}$, A. Gutwillig ${ }^{1}$, Y. Wine ${ }^{2}$, C. Saperia ${ }^{1}$, L. Farhat ${ }^{1}$, L. Tal ${ }^{1}$, H. Gutman ${ }^{1}$, A. Tsivian L. ${ }^{3}$, R. Brenner ${ }^{4}$, E. Abu Bandora ${ }^{1}$, N. Reticker-Flynn ${ }^{5}$, P. Rider ${ }^{1}$, Y. Carmi ${ }^{1}$

${ }^{1}$ Tel Aviv University, Pathology, Tel Aviv, Israel ${ }^{2}$ Tel Aviv University, Molecular Microbiology and Biotechnology, Tel Aviv, Israel ${ }^{3}$ The Edith Wolfson Medical Center, Department of Urologic Surgery, Holon, Israel ${ }^{4}$ The Edith Wolfson Medical Center, Gastrointestinal Oncology Clinic, Holon, Israel

${ }^{5}$ Massachusetts Institute of Technology, Pathology,

Massachusetts, United States Minor Outlying Island

While CD4+ cells are known to associate with improved tumor prognosis, their main role is attributed to supporting the cytotoxic activity of CD8+ T cells and macrophages. By attempting to potentiate antibody-driven immunity, we found a remarkable synergy between CD4+ T cells and tumor-binding antibodies. This surprising synergy was mediated by a small subset of tumor-infiltrating CD4+ T cells express the high affinity $\mathrm{Fc} \gamma \mathrm{R}$ for $\mathrm{IgG}(\mathrm{Fc} \gamma \mathrm{RI})$ in both mouse and human patients. These cells efficiently lyse tumor cells coated with antibodies through concomitant crosslinking of their $\mathrm{T}$ cell receptor (TCR) and Fc $\gamma$ RI. By infecting conventional CD4+ T with $\mathrm{Fc} \gamma \mathrm{RI}$ and its signaling chain, we successfully employed this mechanism to treat established solid cancers. Overall, this discovery shed new light on the biology of this previously unknown $\mathrm{T}$ cell subset, their function during tumor immunity and open a new venue to utilize their unique killing signals in immunotherapy.

\section{ICLEa19-0045}

Fighting Infections and Autoimmune Diseases

\section{CD4+CD25+CD127HI CELLS CORRELATE WITH LENGTH OF REMISSION IN TYPE 1 DIABETES: VALIDATED IN A MULTI-SITE STUDY}
A. Narsale ${ }^{1}$, R. Moya ${ }^{1}$, H. Robertson ${ }^{1}$, D. Payne ${ }^{1}$, T.T. Lu ${ }^{2}$, R. Geoffery ${ }^{3}$, K. Buchanan ${ }^{4}$, M. Harris ${ }^{5}$, A.S. Bergot ${ }^{5}$, R. Thomas ${ }^{5}$, M. Hessner ${ }^{6}$, M. Battaglia ${ }^{7}$, E. Serti ${ }^{8}$, J. Davies $^{1}$

${ }^{1}$ San Diego Biomedical Research Institute, Davies Lab, San Diego, USA ${ }^{2}$ Immune Tolerance Network, Immune Tolerance Network, Washington DC, USA ${ }^{3}$ The Medical College of Wisconson, Dept of Pediatrics, Milwaukee, USA ${ }^{4}$ University of Queensland, Diamantina Institute-, Woolloongabba, Australia ${ }^{5}$ University of Queensland, Diamantina Institute, Woolloongabba, Australia ${ }^{6}$ The Medical College of Wisconsin, Department of Pediatrics, Milwaukee, USA ${ }^{7}$ Diabetes Research Institute DRI, IRCCS San Raffaele Scientific Institute, Segrate, Italy ${ }^{8}$ Immune Tolerance Network, Biomarker and Discovery, Seattle, USA

We have previously shown that the frequency of $\mathrm{CD}^{+}$ $\mathrm{CD} 25^{+} \mathrm{CD} 127^{\mathrm{hi}}$ cells correlates with length of partial remission in children with Type 1 Diabetes (T1D). In healthy adults these cells have a strong Th2 bias and a dominant central memory (CM) phenotype. The purpose of this study was to validate the correlation between $\mathrm{CD} 25^{+} \mathrm{CD} 127^{\text {hi }}$ cells and length of remission in a larger cohort of patients and assess their response to Alefacept in the TIDAL study. In a cohort of 91 T1D patients, a significant correlation is observed between the frequency of $\mathrm{CD} 25^{+} \mathrm{CD} 127^{\mathrm{hi}}$ cells at diagnosis and length of remission. $\mathrm{CD} 25^{+} \mathrm{CD} 127^{\text {hi }}$ cells in T1D express CD2 and are a mix of CM and effector memory (EM) cells, suggesting that they might be a target for Alefacept. Consistent with this notion, the relative frequency of CD25+CD127hi cells is significantly lower at all time points post baseline in Alefacepttreated patients compared to the Placebo group following similar kinetics to that previously reported for $\mathrm{CM}$ and EM cells in the TIDAL study. However, the frequency of baseline $\mathrm{CD} 25^{+} \mathrm{CD} 127^{\mathrm{hi}}$ cells maintained a positive association with length of remission in the Alefacept-treated group, suggesting a role in protection. Possible explanations for the apparent paradox between the capacity of Alefacept to deplete $\mathrm{CD} 25^{+} \mathrm{CD} 127^{\mathrm{hi}}$ cells while maintaining a positive association between $\mathrm{CD} 25^{+} \mathrm{CD} 127^{\mathrm{hi}}$ cell frequency and a longer length of remission are discussed.

\section{ICLEi19-0005}

Fighting Infections and Autoimmune Diseases

\section{IMPROVED CONTROL OF ACTIVATION AND EXPANSION OF TREGS FOR USE IN CELL THERAPY APPLICATIONS}

I.C. Schrøder ${ }^{1}$, K. Bernström ${ }^{1}$, N. Lieske ${ }^{1}$, D. Gjølberg ${ }^{1}$, G. Økern ${ }^{1}$, H. Zhang ${ }^{1}$, T.H. Hereng ${ }^{1}$, U. Rehn ${ }^{2}$, Ø. Åmellem ${ }^{3}$

${ }^{1}$ Thermo Fisher Scientific, Research and development- Cell Medicine, Oslo, Norway ${ }^{2}$ Thermo Fisher Scientific, Cell Culture and Cell Therapy, New York, USA ${ }^{3}$ Thermo Fisher Scientific, Cell Culture and Cell Therapy, Oslo, Norway

Our studies demonstrate that our new CTS ${ }^{\mathrm{TM}}$ Dynabeads ${ }^{\mathrm{TM}}$ Treg Xpander results in superior Treg expansion with maintenance of high \%FOXP3 expression after 12-14 days. The expanded Tregs have suppressive activity and display the characteristic Treg cytokine profile and phenotype. The stability of the expanded Tregs is high, based on analysis of the Tregspecific demethylated region.

Depending on the media, non-serum supplement and growth factors as well as the bead to cell ratio, the protocol can be altered to give a controllable drug of Tregs. The beads are easily removed by CTS ${ }^{\text {TM }}$ DynaMag.

CTS $^{\text {TM}}$ Dynabeads ${ }^{\mathrm{TM}}$ Treg Xpander support consistent and scalable Treg manufacturing for immunotherapies, supplementing our Cell Therapy System $\left(\mathrm{CTS}^{\mathrm{TM}}\right)$ portfolio. 


\section{ICLEa19-0007}

Hematological Malignancies

\section{ADULT PERIPHERAL BLOOD AND UMBILICAL CORD BLOOD NK CELLS ARE GOOD SOURCES FOR EFFECTIVE CAR THERAPY AGAINST CD19 POSITIVE LEUKEMIC CELLS}

L. Herrera $^{1}$, S. Santos ${ }^{1}$, M.A. Vesga ${ }^{1}$, J. Anguita ${ }^{2}$, I. Martin-Ruiz ${ }^{2}$, T. Carrascosa ${ }^{3}$, M. Juan ${ }^{4}$, C. Eguizabal $^{1}$

${ }^{1}$ Osakidetza- Basque Center for Blood Transfusion and Human Tissues, Cell Therapy and Stem Cells Group, Galdakao- 48960Spain, Spain ${ }^{2}$ CIC bioGUNE, Macrophage and Tick Vaccine Laboratory, Derio- Biscay, Spain ${ }^{3}$ Osakidetza-Hospital Galdakao-Usansolo, Servicio de Hematología, Galdakao, Spain ${ }^{4}$ Hospital Clínic de Barcelona. Hospital Sant Joan de Déu. Institut d'Investigacions Biomèdiques August Pi i Sunyer Hospital. Universitat de Barcelona, Servei d'Immunologia, Barcelona, Spain

Background: B-cell hematologic cancers such as leukemia and lymphoma are common forms of pediatric and adult cancers worldwide. Acute Lymphoblastic Leukemia (ALL) is the most common cancer among children with a prevalence of $20-25 \%$ of all cases. The survival rate for these patients at 5 years is $79.2 \%$, but it is still an incurable disease in many patients. Chimeric Antigen Receptor (CAR) T cells therapy has arisen as a new alternative to conventional therapies in order to treat advanced refractory cancers. However, this therapy has some undesirable side effects such as cytokine release syndrome and neurotoxicity. NK cells could be a good alternative for CAR based therapy, as they do not cause these side effects.

Objective: The aim of this study is to compare adult peripheral blood $(\mathrm{AB}) \mathrm{NK}$ cells and cord blood $(\mathrm{CB}) \mathrm{NK}$ cells in order to establish the best one to be used against hematological cancer using CAR technology.

\section{Material and Methods:}

1. Isolation and culture of $\mathrm{NK}$ cells from $\mathrm{AB}$ and $\mathrm{CB}$ samples.

2. Determining CD19-CAR transduction by Flow Cytometry analysis.

3. Cytotoxicity and degranulation assays.

\section{Results:}

- The mean AB CAR-NK transduction efficiency was 47.46 while CB CAR-NK transduction efficiency was 46.8 .

- When exposed to CD19 positive cells, AB CD19-CAR NK cells showed a significantly higher degranulation than nontransduced $\mathrm{AB}$ NK cells. We observed similar behavior with CB CD19-CAR NK cells and non-transduced CB NK cells.

- AB and CB CD19-CAR NK cells lysed better CD19 expressing target cells such as Nalm-6, ALL cells and CLL cells than nontransduced NK cells.

\section{Conclusions:}

1. We are capable of infecting $\mathrm{AB}$ and $\mathrm{CB}$ NK cells with CD19-CAR.

2. CD19-CAR infected NK cells kill more effectively CD19 expressing target cells than those which are not infected.

3. CB CD19-CAR infected NK cells and AB CD19-CAR infected NK cells are similar at killing.

4. Storage cord blood could be used for CAR therapies.
ICLEa19-0026

New Horizons in Lympocyte Engineering

GENETIC MODIFICATION OF CD8+ T CELLS TO EXPRESS EGFR: POTENTIAL APPLICATION FOR ADOPTIVE T CELL THERAPIES

N. Casares ${ }^{1}$, J.J. Lasarte ${ }^{1}$, T. Lozano ${ }^{1}$, S. Chocarro ${ }^{1}$, C. del Valle ${ }^{1}$, M. Gorraiz ${ }^{1}$, S. Hervas-Stubbs ${ }^{1}$, P. Sarobe ${ }^{1}$

${ }^{1}$ Center for Applied Medical Research CIMA, Immunology and Immunotherapy Program, Pamplona, Spain

Adoptive immunotherapy with ex vivo-expanded tumorinfiltrating lymphocytes (TILs) has achieved objective clinical responses in a significant number of patients with cancer. However, the failure of many patients to develop long-term tumor control may be, in part, due to exhaustion of transferred $\mathrm{T}$ cells in the presence of a hostile tumor microenvironment. In several tumor types, growth and survival of carcinoma cells appear to be sustained by a network of receptors/ligands of the ErbB family. We wondered if transferred $\mathrm{T}$ cells could benefit from cytokines produced into the tumor, such as ligands for EGFR, in order to proliferate better and exert their anti-tumor activities more efficiently. For this purpose, we generated a retroviral vector for transducing $\mathrm{T}$ lymphocytes to express functional EGFR. In vitro, EGFRexpressing CD8 T cells from OT1 mice proliferated better and produced more IFNg and TNFa in the presence of EGFR ligands produced by tumor cells. Moreover, these modified $\mathrm{T}$ cells were able to recognize and lyse B16.OVA cell line. Importantly, EGFRengineered CD8 lymphocytes adoptively transferred had a better therapeutic effect on tumor bearing mice showing a significant delay in tumor growth. Our results suggest that genetic modification of CD8 T cells to express EGFR might be considered in immunotherapeutic strategies based on adoptive transfer of $\mathrm{T}$ cells against cancers expressing EGFR ligands.

ICLEa19-0008

New Horizons in Lympocyte Engineering

UNDERSTANDING THE EXPRESSION PROFILE OF NKG2D LIGANDS ON T CELLS: IMPLICATIONS FOR CAR T CELL THERAPY

B. Demoulin $^{1}$, E. Breman ${ }^{1}$, S. Bornschein ${ }^{1}$, M. Fontaine ${ }^{1}$, P. Sotiropoulou A. ${ }^{1}$, D. Gilham ${ }^{1}$

${ }^{1}$ Celyad, Research and development, Mont-Saint-Guibert, Belgium

Background: CYAD-01 is a T cell product engineered to express a NKG2D-based chimeric antigen receptor (CAR) that binds to eight stress-induced ligands (MICA, MICB and ULBP1-6) found on a broad range of haematological malignancies and solid tumours. Phase I Clinical trial testing CYAD-01 safety, already displayed promising results in haematological malignancies (THINK trial). Yet, T cells can upregulate NKG2DL expression during mitogenic activation which may result in self killing driven by the CAR (fratricide). In order to understand this phenomenon, the expression profile and kinetics of expression of NKG2D ligands (NKG2DL) during the CYAD-01 CAR T cell manufacturing process and target cell engagement was investigated.

Methods: NKG2DL expression on CYAD-01 was determined by flow cytometric analysis at different stages of $\mathrm{T}$ cell manufacture. NKG2DL expression was also determined on CYAD-01 cells and target cells during effector activation. The specificity of CYAD-01 for individual NKG2DL was determined by coating 
purified protein ligands onto culture wells and determining cytokine response during culture.

Results: MICA and MICB were the two predominant NKG2DL shown to be upregulated on CYAD-01 cells during manufacture and during effector engagement with target. ULBP1 expression was observed less frequently. While all purified NKG2DL induced a functional response by CYAD-01 cells, there was a clear preferential activation induced by MICA and MICB, which could induce cytokine production by CYAD-01 cells at 10fold lower concentration compared to other NKG2DL.

Conclusion: Given that MICA and MICB are the main NKG2DL expressed on CYAD-01 cells upon activation and also are the predominant targets of the NKG2D CAR, the knockdown of MICA and MICB expression on CYAD-01 cells seems to be the most effective way to inhibit fratricide. Further studies will investigate the specific knockdown of MICA and MICB and determine the impact on CYAD-01 $\mathrm{T}$ cell anti-tumor activity in vitro and in vivo.

\section{ICLEa19-0006}

New Horizons in Lympocyte Engineering

\section{ESTABLISHING NOVEL RECEPTOR-TARGETED LENTIVIRAL VECTORS FOR T-LYMPHOCYTE- DIRECTED IN VIVO GENE DELIVERY}

A. Frank $^{1}$, F.B. Thalheimer ${ }^{2}$, W. Uckert $^{3}$, J. Hartmann $^{1}$, C.J. Buchholz ${ }^{1}$

${ }^{1}$ Paul-Ehrlich-Institut, Division of Medical Biotechnology, Langen, Germany ${ }^{2}$ Paul-Ehrlich-Institut, Molecular Biotechnology and Gene Therapy, Langen, Germany ${ }^{3}$ Max-Delbrück-Center for Molecular Medicine in the Helmholtz. Association, Molecular Cell Biology and Gene Therapy, Berlin, Germany

Undoubtfully, receptor-targeted lentiviral vectors (LVs) have been established as promising tools for precise genetic modification of distinct cellular subtypes in vitro and in vivo. The recent success of a CD8-targeted LV in generating cytotoxic chimeric antigen receptor (CAR) T cells directly in vivo shows the power of this technology and strongly supports further developments especially with regard to $T$ cell manipulation. Here, we generated novel potent $T$ cell-targeted lentiviral vectors delivering genes exclusively and specifically into both human and non-human primate (NHP) T lymphocytes. The targeting domain of these LVs consisted of either the conventionally used antibody-derived single-chain variable fragments, or designed ankyrin repeat proteins (DARPins). Besides selective $\mathrm{T}$ cell attachment, binders mediating T cell activation may be of special interest for in vivo gene delivery. DARPins were selected to bind to human and NHP cytotoxic T cells by screening of a combinatorial DARPin library by ribosome display using recombinant extracellular domains of target receptors expressed in human cells as bait. Selectivity of binders was confirmed by receptor binding analysis on primary human and NHP cells. All generated novel T cell-targeted LVs were exquisitely specific for their target cell population, yet highly active in gene delivery into primary human and NHP cells. In vivo, the vectors showed comparable or even more efficient $T$ cell-specific transduction than the benchmark CD8LV when equal particle numbers were applied. Importantly, the novel LVs selectively transduced primary NHP T lymphocytes, thereby offering the opportunity to analyze their specificity and potency in large animal models such as non-human primates.

\section{ICLEa19-0032}

New Horizons in Lympocyte Engineering

\section{A LIPOCALIN-BASED ON-SWITCH FOR CONTROLLING CAR T CELLS WITH AN ORALLY AVAILABLE DRUG}

C. Brey ${ }^{1}$, M. Dobersberger ${ }^{1}$, I. Schaffner ${ }^{2}$, G. Mlynek ${ }^{3}$, D. Pühringer ${ }^{3}$, B. Salzer ${ }^{1}$, K. Djinovic-Carugo ${ }^{3}$, C. Obinger ${ }^{4}$, W. Holter ${ }^{5}$, M. Traxlmayr ${ }^{4}$, M. Lehner ${ }^{1}$

${ }^{1}$ Children's Cancer Research Institute, Development of Cellular Therapeutics, Vienna, Austria ${ }^{2}$ BOKU-University of Natural Resources and Life Sciences, Core Facility for Biomolecular \& Cellular Analysis, Vienna, Austria ${ }^{3}$ University of Vienna, Department for Structural and Computational Biology- Max F. Perutz Laboratories, Vienna, Austria ${ }^{4}$ BOKU-University of Natural Resources and Life Sciences, Department of Chemistry, Vienna, Austria ${ }^{5}$ Medical University of Vienna, St. Anna Kinderspital, Vienna, Austria

The regulation of a protein-protein interaction with a small molecule represents a molecular $\mathrm{ON}$-switch and enables the control of cellular functions. Most current ON-switches for conditional protein-heterodimerization lack essential prerequisites for therapeutic application, such as the usage of human protein components and small molecules suited for clinical application.

Here we report the development of an allosteric ON-switch system which is based on a human protein and can be regulated by an orally applicable drug. This allosteric ON-switch system relies on the selection of binders which recognize conformational changes induced in a small molecule specific manner in a lipocalin protein. Human lipocalins have a deep binding pocket surrounded by flexible loops and have evolved for binding of a multitude of diverse small molecules. For generating a prototypic ON-switch we chose the human lipocalin retinol binding protein-4 (hRBP4) and the orally applicable drug A1120. With this system, we could engineer two different binder scaffolds which specifically recognize the A1120-induced conformation of hRBP4. Integration of this hRBP4-based ON-switch system into a chimeric antigen receptor (CAR) enabled efficient regulation of CAR-T cell activity.

Our molecular ON-switch system combines the benefits of a non-toxic, orally applicable drug with human protein components (hRBP4) and has the potential to be broadly applied for the control of cellular therapeutics such as CAR-T cells.

\section{ICLEa19-0047}

New Horizons in Lympocyte Engineering

\section{ENGINEERING T CELLS AND B CELLS FOR IMMUNOTHERAPY USING V(D)J RECOMBINATION}

\section{Nataf $^{1}$, I. Dotan $^{1}$, M. Horovitz-Fried ${ }^{1}$, N. Gritsenko ${ }^{1}$, C. Pundak-Mintz ${ }^{1}$, A. Nahmad ${ }^{1}$, A. Barzel ${ }^{1}$ \\ ${ }^{1}$ Tel Aviv University, School of Neurobiology-Biochemistry \& Biophysics, Tel Aviv, Israel}

T cell engineering for immunotherapy has shown clinical success, but large scale application is hindered by reliance on cumbersome ex vivo manipulations. In addition, B cell engineering has not shown therapeutic efficacy to date. Conversely, recombinant Adeno Associated vectors (rAAV) allow in vivo lymphocyte transduction but are seldom used for immunotherapy as they rarely integrate for stable expression in dividing cells. Here, we propose a 
novel immunotherapy approach -“"VDJ targeting",": targeting rAAV-delivered immune genes into the genome using $\mathrm{V}(\mathrm{D}) \mathrm{J}$ recombination in developing lymphocytes. A receptor/Ab gene flanked by recognition signal sequences (RSS) is inserted into the endogenous locus by the recombination activating gene (RAG) complex during $\mathrm{V}$ (D)J recombination. In particular, in the $\mathrm{T}$ lineage, we target chimeric antigen receptor (CAR) or T cell receptor (TCR) genes into loci coding TCR chains and, in the B lineage, we target antibody $(\mathrm{Ab})$ genes into loci coding Ab chains. Here, we demonstrate VDJ targeting in the B and T lineages in immortalized and inducibly differentiating cells as well as in in vitro differentiating primary lymphocytes and in fresh murine bone marrow. Next, we aim to demonstrate the therapeutic application of lymphocyte engineered by VDJ targeting. VDJ targeting may have several advantages over state of the art technologies: Only developing lymphocytes, expressing RAG, incorporate the receptor/Ab gene, which is subsequently expressed in potent naïve cells. Targeted $\mathrm{T}$ cells may express only the desired receptors, due to allelic exclusion, while targeted B cells may express the transgene as a B cell receptor, and upon activation undergo affinity maturation allowing potent immune response, memory retention and diminished antigenic escape. VDJ targeting may allow safe, efficient and scalable engineering of $\mathrm{B}$ cells and $\mathrm{T}$ cells, both in vivo and ex vivo.

\section{ICLEa19-0016}

Non-Viral Vectors and Transposons

\section{VAPOR NANOBUBBLE PHOTOPORATION AS A NEW, SAFE AND EFFICIENT TECHNIQUE FOR THE DELIVERY OF SMALL INTERFERING RNA TO LYMPHOCYTE CELLS}

\author{
J. Van Hoeck ${ }^{1}$, L. Wayteck ${ }^{1}$, R. Xiong ${ }^{1}$, K. Braeckmans ${ }^{1}$, \\ S.C. De Smedt ${ }^{1}$, K. Raemdonck ${ }^{1}$ \\ ${ }^{1}$ Ghent University, Faculty of Pharmaceutical Sciences, Ghent, \\ Belgium
}

The adoptive transfer of cytotoxic T lymphocytes (CTLs) and natural killer cells $(\mathrm{NK})$ has shown great clinical success in treatment of blood cancers and melanoma. However, treatment of solid tumors remains difficult due to their immunosuppressive microenvironment. Using siRNA therapy, we aim to increase the effectiveness of transferred CTLs and NK cells in patients with solid tumors. This by selectively down-regulating immunosuppressive pathways prior too transfusion, increasing the transferred cells' resistance against immunosuppressive signals at the tumor site. As such we aim to increase the lymphocyte's capacity to infiltrate tumors, proliferate and eradicate tumor cells. Unfortunately, it is well known that these primary cells are hard-to-transfect and conventional non-viral transfection agents are generally ineffective. Viral transduction and electroporation are more efficient but their use is restricted by high cost, safety issues, and cytotoxicity. Photoporation has recently gained interest as a more gentle alternative physical approach to deliver membrane-impermeable macromolecules into cells. By attaching gold nanoparticles to the cell surface followed by pulsed laser illumination, transient membrane pores can be generated that allow the diffusion of macromolecules directly into the cell cytosol. Here, we evaluated this technique for the non-toxic and effective delivery of small interfering RNA (siRNA) and subsequent silencing of target genes in activated CTLs (1) and human NK cells. We compared photoporation with nucleofection, the current standard physical technique for lymphocyte transfection, and demonstrated a significantly reduced cytotoxicity and higher average dose per cell for the photoporation technique.
(1) Wayteck L., Xiong R., Braeckmans K., De Smedt S. C., Raemdonck K. (2017).Comparing photoporation and nucleofection for delivery of small interfering RNA to cytotoxic T cells. J. Control. Release 267, 154-162.

\section{ICLEa19-0040}

Other

\section{DIRECT TRANSGENE EXPRESSION AND MAB PRODUCTION BY USING NEW GENERATION MINIMISED UCOES ON HUMAN IPS AND CHO CELLS}

\author{
O. Anakok ${ }^{1}$ \\ ${ }^{1}$ Ataturk University, Molecular Biology \\ and Genetics, Erzurum, Turkey
}

It has recently been shown that efficient and stable expression of transgenes from the A2UCOE is at least in part due to its resistance to DNA methylation-mediated silencing. Analysis of a deletion series from the CBX3 end of a fully functional $2.2 \mathrm{~kb}$ A2UCOE where expression of an eGFP reporter gene is directly driven off the HNRPA2B1 promoter, revealed a $1.2 \mathrm{~kb}$ and $1.7 \mathrm{~kb}$ truncation that retained full UCOE activity following transduction of P19 and F9 cells in both undifferentiated and differentiated states. These 1.2-1.7 and a further deletion $0.5 \mathrm{~kb}$ A2UCOEs, were also able to retain stable expression in murine embryonic stem cells and now in human iPS cells during differentiation into embryoid bodies and tissue specific cell types. In addition, from a practical perspective our finding that the 0.5-1.2-1.7A2UCOEs retain the same stability of expression as the larger 2.2A2UCOE patent suggests that it can effectively replace the latter within therapeutic LV constructs allowing a greater capacity and also more safety for the gene of interest by cutting off the enhancer elements which carries a potential mutation risk. A further project now on human $\mathrm{mAb}$ production with recombinant $\mathrm{CHO}$ cells by using our new UCOE models has been started and the early results show that they are more productive than the control A2UCOEs and able to produce human recombinant $\mathrm{mAbs}$ in $\mathrm{mg}$ to $\mathrm{g}$ level even under two months of cultivation. The results already encouraging to provide a new better UCOE model for recombinant protein production in mammalian cells.

\section{ICLEi19-0009}

\section{Other}

\section{RECOMBINANT GROWTH FACTOR RECEPTORS ENHANCE T-CELL PROLIFERATION AND ANTI- TUMOUR ACTIVITY IN A MODEL OF ADOPTIVE T-CELL THERAPY}

M. Le Brocq ${ }^{1}$, T. Katopodi ${ }^{1}$, M. Sykorova ${ }^{1}$, H. Crookes ${ }^{1}$, N. Hurst ${ }^{2}$, R. Hawkins ${ }^{1}$, G. Kueberuwa ${ }^{1}$, J. Bridgeman ${ }^{1}$

${ }^{1}$ Immetacyte Ltd., Research \& Development, Manchester, United Kingdom ${ }^{2}$ University of Leeds, Biological Sciences, Leeds, United Kingdom

T-cells isolated from tumour biopsies can be expanded ex vivo to huge numbers before readministration to patients whereupon they impart therapeutic benefit. So-called Tumour infiltrating lymphocyte (TIL) therapy has proved hugely successful in numerous clinical trials for treatment of metastatic melanoma, and have also shown benefit in other indications. Preconditioning chemotherapy and post-infusion IL-2 are currently used to potentiate TIL survival and engraftment but are associated 
with significant toxicity which in turn impacts on the patients well-being, the infrastructure required to deliver cell therapies and the overall cost of treatment.

We have explored the possibility of generating a synthetic recombinant growth factor receptor (rGFR) which can be controlled via exogenous administration of a clinically available drug. To this end we have based our rGFR on the thrombopoietin receptor (TpoR) which is normally expressed in megakaryocytes where it controls differentiation and platelet production. T-cells engineered with this rGFR respond to thrombopoietin and the mimetic drug Eltrombopag.

Treatment of T-cells with Eltrombopag results in enrichment of engineered cells when cultured alone, furthermore, we observed enhanced survival of engineered melanoma and ovarian TIL when mixed with their matched autologous tumour lines. We have developed the basic receptor format to derive an optimised receptor with enhanced activity, for which we have identified a biochemical basis based on pSTAT status. We have also developed a method to deliver these rGFRs to TIL at high efficiency and in a GMP compliant manner which will expedite their translation into a manufacturing process for clinical trials.

\section{ICLEa19-0018 \\ Other}

\section{IMPLICATION OF TRPV1 IN TEMPERATURE AND PH-SENSING BY CANINE PBMC}

$\underline{\text { J. Bujak }}^{1}$, D. Kosmala ${ }^{1}$, A. Nyc ${ }^{1}$, I. Szopa ${ }^{1}$, K. Majchrzak ${ }^{1}$, P. Bednarczyk ${ }^{2}$

${ }^{1}$ Warsaw University of Life Sciences - SGGW, Department of Physiological Sciences, Warsaw, Poland ${ }^{2}$ Warsaw University of Life Sciences - SGGW, Department of Biophysics, Warsaw, Poland

TRP ion channels are involved in calcium signaling, necessary for induction of cell proliferation, differentiation and transcriptional gene regulation. Distinct members of TRP family are sensitive to a range of physical and chemical stimuli including temperature, $\mathrm{pH}$ and phytochemical. Thermo- and $\mathrm{pH}$-sensitivity along with contribution to calcium signaling makes TRPs potential regulators of the immune response. Among TRP ion channels, TRPV1, known as capsaicin receptor, is sensitive to temperature and external $\mathrm{H}^{+}$. Its activity was also shown to be influenced by proinflammatory cytokines such as IL- $1 \beta$, IL- 6 and TNF- $\alpha$. Therefore, TRPV1 seems to be a good candidate for modulation of the immune cell functions.

The main objectives of the study were 1) to determine how the temperature and $\mathrm{pH}$ influence activation of the canine T cells, 2) to evaluate the functionality of the TRPV1 expressed by the canine PBMCs, 3) to assess whether TRPV1 is involved in immune cells-microenvironment crosstalk.

Expression of TRPV1 in the canine PBMC was confirmed on both mRNA and protein level. Patch clamp recording revealed the existence of capsaicin-sensitive current in the canine PBMC. Furthermore, the increase in intracellular $\mathrm{Ca}^{2+}$ was observed when PBMCs were exposed to either increase in temperature or to capsaicin. Low $\mathrm{pH}$ (6.0) caused significant decrease in the $\mathrm{T}$ cell activation, however it was not associated with the decrease of intracellular $\mathrm{pH}$. Presence of thermo- and $\mathrm{pH}$-sensitive receptors might partially explain the impact of these factors on the immune cells functioning.
The research was supported by National Science Centre Grant 2016/21/N/NZ6/02137 and First TEAM programme of the Foundation for Polish Science co-financed by European Union under the European Regional Development Fund (POIR.04.04.0000-3EE9/17-00).

\section{ICLEa19-0025}

Other

\section{EFFECTOR CD4+ T CELLS, RATHER THAN CD8+, INCREASE THE EFFICACY OF ANTIBODY-DRIVEN TUMOR IMMUNITY}

\author{
A. Gutwillig ${ }^{1}$, D. Rasoulouniriana ${ }^{1}$, N. Santana-Magal ${ }^{1}$, \\ L. Farhat ${ }^{1}$, L. Tal ${ }^{1}$, P. Rider ${ }^{1}$, Y. carmi ${ }^{1}$ \\ ${ }^{1}$ Tel-Aviv University, pathology, Tel Aviv, Israel
}

Most studies and therapeutic strategies in tumor immunology have focused on $\mathrm{CD}^{+} \mathrm{T}$ cells and their cytotoxic activity, with relatively little emphasis has been placed on understanding and harnessing the $\mathrm{CD}^{+} \mathrm{T}$ cell response. In a recent study, aimed to dissect the changes in $\mathrm{T}$ cell compartment across the body of tumor-bearing mice following antibody-mediated immunotherapy, we found that antigen-experienced $\mathrm{CD}^{+}$and $\mathrm{CD}^{+} \mathrm{T}$ cells are highly associated with tumor regression. Here, we further tested which cell subset better potentiate antibody-mediated tumor immunity. Initially, we isolated effector $\mathrm{CD}^{+}$and $\mathrm{CD}^{+} \mathrm{T}$ cells from tumor-bearing mice, expend them ex vivo and inject them intravenously to mice bearing established melanoma tumors along with tumor-binding antibodies. We found that adoptive transfer of effector $\mathrm{CD} 4^{+} \mathrm{T}$ cell induced a longer, more potent tumor-protecting immunity compared to transfer of effector $\mathrm{CD}^{+} \mathrm{T}$ cells. Deep sequence analysis of $\mathrm{CD}^{+} \mathrm{T}$ cell receptors (TCR) in tumor-bearing mice revealed that $\mathrm{T}$ cell in different organs, and especially in tumors, have different TCR. Therefore, we sought to elucidate which organ contains the most effective tumor-reactive $\mathrm{CD} 4^{+} \mathrm{T}$ cells. To this end, we isolated effector $\mathrm{CD}^{+} \mathrm{T}$ cells from various organs of tumor-bearing mice, expend them ex vivo and adoptively transferred them to mice bearing large and established melanoma. We found that $\mathrm{CD}^{+} \mathrm{T}$ cells from tumors and draining lymph nodes, but not from blood or non-draining lymph nodes, induce regression of large established tumors. Overall, this study highlights the therapeutic capacities of $\mathrm{CD}^{+} \mathrm{T}$ cells and their synergism with antibody-driven immunotherapies, and provides a rationale for deeper studies aimed at leveraging $\mathrm{CD}^{+} \mathrm{T}$ cell responses.

\section{ICLEa19-0022 \\ Other}

\section{PROGRAMMING OF CD4+ T LYMPHOCYTES TOWARD A TH17 PHENOTYPE - IN VITRO STUDY ON COMPANION DOG MODEL}

\author{
I. Szopa $^{1}$, J. Bujak ${ }^{1}$, M. Granica ${ }^{1}$, K. Majchrzak ${ }^{1}$ \\ ${ }^{1}$ Warsaw University of Life Sciences - Faculty of Veterinary \\ Medicine, Department of Physiological Sciences, Warsaw, \\ Poland
}

Th17 lymphocytes have been shown to efficiently eradicate large established melanoma tumors upon adoptive cell transfer (ACT) therapy on mouse model. These cells are characterized by production of IL-17 and expression of retinoic acid-related orphan receptor $(\mathrm{ROR} \gamma \mathrm{t})$. Moreover, Th17 cells are resistant to 
apoptosis and exhibit stem cell-like properties, thus retain potent antitumor activity after long-term ex vivo expansion.

The aim of the study was to determine activation and expansion protocol for canine $\mathrm{CD} 4^{+} \mathrm{T}$ lymphocytes as well as their programing toward Th17 phenotype. Furthermore, modulation of intrinsic signaling pathways of canine $\mathrm{T}$ cells in order to support Th17 differentiation.

We isolated $\mathrm{T}$ lymphocytes from peripheral blood of domestic dogs (Canis lupus familiaris) and activated them using either Concanavalin A (natural mitogen) or epoxylated magnetic beads coated with anti-canine CD3 and CD28 antibodies. For type 17 polarization canine IL- $1 \beta$, IL-6, TGF- $\beta$, anti-IL-4 and anti-IFN- $\gamma$ antibodies were used. Moreover, the effect of indomethacin (blocker of $\beta$-catenin expression) on the Th17 programing was examined.

Our research have shown increased CD25 expression and IL-2 production by canine $\mathrm{CD}^{+} \mathrm{T}$ cells as well as morphological changes during blast transformation upon activation using epoxylated magnetic beads. Moreover, we programmed canine Th17 lymphocytes, which exhibited high ROR $\gamma \mathrm{t}$ expression and released significant amount of IL-17. Our study revealed that administration of indomethacin further enhanced IL-17 production (up to 55\%) and up-regulated expression of Th17 cell-associated genes.

Overall our research determined the optimal conditions for canine Th17 cells expansion for further immunological assessment and paved the way for tumor-bearing dog studies of ACT therapy with Th17 cells, that might benefit and facilitate design of human clinical trials.

The project is carried out within the First TEAM programme of the Foundation for Polish Science co-financed by European Union under the European Regional Development Fund (POIR.04.04.00-00-3EE9/17-00).

\section{ICLEi19-0006}

\section{TCR Targeting Solid Tumors}

\section{GMP MANUFACTURING OF TCR-ENGINEERED T CELLS IN 5-6 DAYS}

M. Kalra ${ }^{1}$, Z. Coughlin ${ }^{1}$, A. Alpert ${ }^{2}$, L. Alten $^{3}$, S. Bunk ${ }^{3}$, C. Wagner ${ }^{3}$, J. Fritsche ${ }^{4}$, O. Schoor ${ }^{4}$, D. Maurer ${ }^{3}$, T. Weinschenk ${ }^{4}$, K. Sieger ${ }^{5}$, A. Mohamed ${ }^{1}$, S. Walter ${ }^{6}$
${ }^{1}$ Immatics US Inc, Chemistry Manufacturing \& Control, Houston, USA ${ }^{2}$ Immatics US Inc, Immunology, Houston, USA ${ }^{3}$ Immatics Biotechnologies GmbH, Immunology, Tuebingen, Germany ${ }^{4}$ Immatics Biotechnologies GmbH, Discovery, Tuebingen, Germany ${ }^{5}$ Immatics US Inc, Quality Control \& Quality Assurance, Houston, USA ${ }^{6}$ Immatics US Inc, Sr Management, Houston, USA

Shorter ex vivo expansion and overall "turnaround time" can have a substantial impact on the quality of T-cell products and their widespread application in cancer immunotherapy. Here, we present development of a shortened GMP manufacturing process with a total of only 5-6 days manufacturing time for a TCRengineered T-cell product (IMA203) under Immatics' ACTengine $^{\circledR}$ (Adoptive Cellular Therapy with autologous engineered $\mathrm{T}$ cells) program. IMA203 targets a novel HLA-A*02:01 restricted tumor peptide antigen identified by Immatics' proprietary XPRESIDENT $^{\circledR}$ platform and validated by XPRESIDENT $^{\circledR}$ guided extensive safety screening.

The manufacturing of ACTengine ${ }^{\circledR}$ products is based on a short and robust process involving activation of $\mathrm{T}$ cells, transduction with a viral vector expressing the transgenic TCR, and expansion in the presence of cytokines. Shorter ex vivo expansion has been shown to improve the phenotype and functionality of $\mathrm{T}$ cells. We observed that the products harvested after a short expansion period exhibited less differentiated memory phenotype with higher proportion of cells expressing CD27 and CD28 costimulatory receptors. Less expanded cells also demonstrated sustained killing of tumor cells in vitro and increased IFN $\gamma$ production in response to cell lines expressing the target antigen at different levels. To keep the yield of TCR-transduced T cells high, the starting T cells (PBMC) are increased up to twofold. Effective measures were taken to overcome practical challenges and mitigate all risks associated with the handling of large number of cells. This process improvement effort resulted in a robust 5-6 day long, semi-closed manufacturing process for IMA203 trial with a "turnaround" time of 19-20 days including 14-day compendial sterility testing. Qualification runs were successfully completed in GMP confirming the feasibility and reproducibility of the new process. Post-IND approval, the process is being currently used for manufacturing 8 patients' products under recently opened IMA203 basket trial (NCT03686124) for treatment of solid tumors. 